\title{
Heavy Metal Levels and Risk Assessment from Consumption of Marine Fish in Peninsular Malaysia
}

\author{
Wan Nurul Farah Wan Azmi*, Nurul Izzah Ahmad, Wan Rozita Wan Mahiyuddin \\ Environmental Health Research Centre, Institute for Medical Research, Ministry of Health, Selangor, Malaysia \\ Email: *nurulfarah@imr.gov.my
}

How to cite this paper: Azmi, W.N.F.W., Ahmad, N.I. and Mahiyuddin, W.R.W. (2019) Heavy Metal Levels and Risk Assessment from Consumption of Marine Fish in Peninsular Malaysia. Journal of Environmental Protection, 10, 1450-1471. https://doi.org/10.4236/jep.2019.1011086

Received: August 22, 2019

Accepted: November 1, 2019

Published: November 4, 2019

Copyright $\odot 2019$ by author(s) and Scientific Research Publishing Inc. This work is licensed under the Creative Commons Attribution International License (CC BY 4.0).

http://creativecommons.org/licenses/by/4.0/

\section{(c) (i) Open Access}

\begin{abstract}
Fish consumption is one of the primary sources of protein in Malaysia. However, harmful substances, including heavy metals released from anthropogenic sources may accumulate in marine organisms through the food chain. Hence, human health risks may occur through the consumption of fish contaminated by heavy metals. This study was conducted to determine the concentrations of heavy metals and to assess health risks in edible tissues of 296 commonly consumed marine fish throughout Peninsular Malaysia. The marine fish samples were collected from selected major fish landing ports throughout Peninsular Malaysia. This paper focused on nine heavy metals concentrations namely selenium $(\mathrm{Se})$, cadmium $(\mathrm{Cd})$, lead $(\mathrm{Pb})$, copper $(\mathrm{Cu})$, zinc $(\mathrm{Zn})$, antimony $(\mathrm{Sb})$, tin $(\mathrm{Sn})$, chromium $(\mathrm{Cr})$ and manganese $(\mathrm{Mn})$ in 46 species of marine fish. The fish samples were digested using a microwave digestion system (Multiwave 3000, Anton Paar). Heavy metals concentrations were analyzed by Inductively Coupled Plasma Mass Spectrometry (ICP-MS) ELAN 9000 (Perkin Elmer, Sciex, Canada). The heavy metals concentrations in marine fish samples were found to be dominated by $\mathrm{Zn}$ followed by $\mathrm{Sn}, \mathrm{Se}$, $\mathrm{Cu}, \mathrm{Mn}, \mathrm{Cr}, \mathrm{Pb}, \mathrm{Cd}$ and $\mathrm{Sb}$ which ranged between $15.9612 \mathrm{mg} / \mathrm{kg}(\mathrm{Zn})$ and $0.0002 \mathrm{mg} / \mathrm{kg}(\mathrm{Sb})$ wet weight. Among the investigated fish species, Otolithoides biauritus demonstrated the lowest concentration for all heavy metal except for $\mathrm{Pb}$. The estimated weekly intakes (EWI) for all samples in this study were below the established PTWI by JECFA of FAO/WHO. Risk assessment results showed that the hazard quotient (HQ) and hazard index (HI) values were lower than 1 in all fish species. The results indicate that exposure to the studied metals poses a low non-carcinogenic risk and considered safe for human consumption.
\end{abstract}

\section{Keywords}

Trace Elements, Hazard Quotient, Hazard Index, ICP-MS, PTWI 


\section{Introduction}

Fish is widely consumed by many people throughout the world because of its high protein content and provides various vitamins, minerals and polyunsaturated omega-3 fatty acids that help reduce the risk of certain types of cancer and cardiovascular diseases [1] [2] [3] [4]. Consumption of fish is a foremost source of protein for the Malaysian population. The Malaysian Adult Nutrition Survey (MANS) reported that the average daily consumption of fish is one and a half of medium size fish per day for the adult population in Malaysia [5] [6]. However, the health benefits provided through fish consumption may be compromised with the presence of contaminants in the fish which can pose a significant threat to the health of consumers [7].

Industrial and urbanisation activities have contributed to the increase of contamination, including heavy metal in the marine environment and have directly influenced coastal ecosystems [8] [9]. The harmful minerals and metals existing in the environment can be absorbed into living organisms from the surrounding water, sediment, and diet. Heavy metals enter the food chain through direct consumption of water or organisms or uptake processes and can be potentially accumulated in fish [10]. Therefore, fish are vulnerable to the effects of chemical contaminants, including heavy metals which bioaccumulate and biomagnify through the aquatic food chain [11]. Feeding habits, size, lifestyle and species of fish may influence metal bioaccumulation ability [12] [13] [14]. The concentrations of heavy metals in the tissues and organs of fishes indicate the concentrations of heavy metals in water, and their accumulation in food chains [4] [15]. The toxic elemental contaminants in fish are transferred into human metabolism through consumption of contaminated fish that could cause unhealthy effects towards human health [16] [17] [18].

Concerning the toxicology effect of heavy metals from fish consumption, the Joint of Food and Agriculture Organization and World Health Organization (FAO/WHO) Expert Committee on Food Additives (JECFA) has set a provisional tolerable weekly intake (PTWI) for protection of the consumer. PTWI is the safe level of intake or the maximum amount of a contaminant that be exposed to a person weekly over a lifetime without an intolerable risk of health effects associated with the consumption of foods [7] [19] [20]. These limits can also be species-specific as metal accumulation is affected by different development and metabolic rates of different organisms [21]. To estimate the potential of health risk due to exposure to the contaminant, the United State Environmental Protection Agency (USEPA), created the reference dose (RfD). RfD is an estimate of daily oral exposure of contaminant to the human population that is likely to be without considerable risk of harmful effects during a lifetime [22].

Numerous studies have investigated heavy metal concentrations in fishes in other parts of Malaysia [9] [11] [23]-[27]. However, most of the studies were focusing on a specific locality, and few studies assessed the potential health effect due to the consumption of fish. Therefore, this study was conducted to deter- 
mine the concentrations of heavy metals and to assess health risks for the edible tissues of 296 commonly consumed marine fish samples from fish landing ports and wholesale markets throughout Peninsular Malaysia. Estimated Weekly Intake (EWI) of elements of fishes was calculated to estimate the risk of consuming contaminated fish. Whereas, the risk of non-carcinogenic effects from fish consumption was assessed by estimating the ratio of the exposure dose to the reference dose from USEPA.

\section{Materials and Methods}

\subsection{Sampling and Sample Preparation}

A total of 394 seafood samples were collected from selected major fish landing ports of Fisheries Development Authority of Malaysia (Lembaga Kemajuan Ikan Malaysia, LKIM) and wholesale markets in Peninsular Malaysia. The sampling was conducted from June to December 2009. This paper reported results for nine heavy metals concentrations, which consisted of 296 numbers of samples included 46 species of marine fish. The selection of fish was based on the most popular/consumed seafood by the local population as described elsewhere [6]. Six major fish landing complexes of LKIM and five wholesale wet markets throughout Peninsular Malaysia were selected as the sampling location. The selected sampling locations are the main fish landing port where fishes were distributed throughout the Peninsular Malaysia, which is represented the whole population consumption of fish (Figure 1). The complete methodology of the sample calculation and collection has been described by Ahmad et al., 2015a [6].

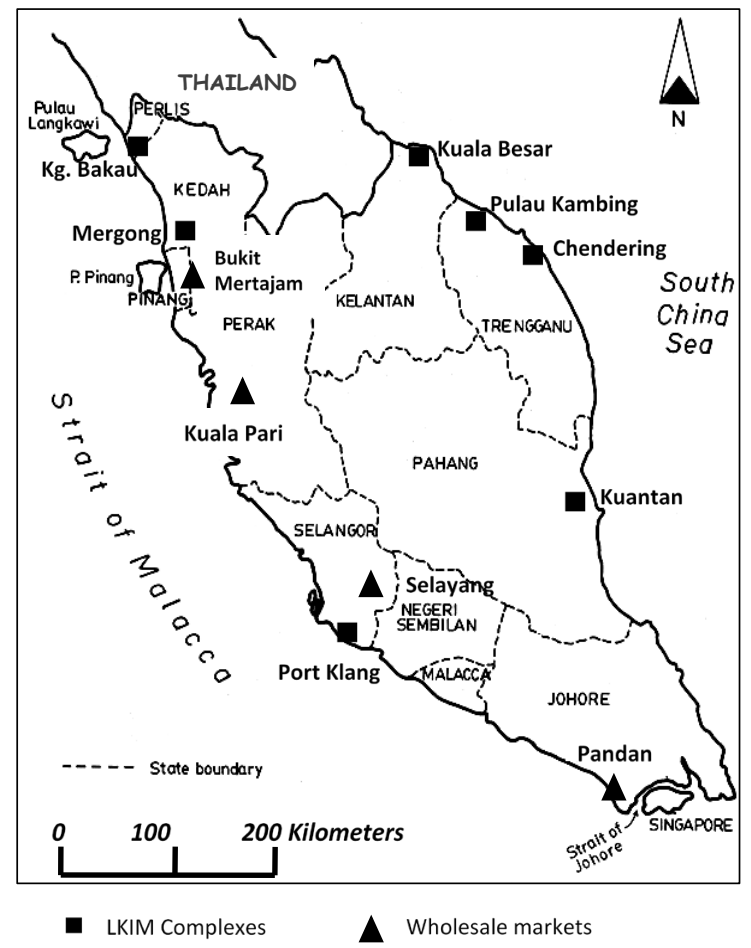

Figure 1. Map of sampling location in Peninsular Malaysia [6]. 


\subsection{Apparatus and Reagent}

All single elements stock solution (selenium, cadmium, lead, copper, zinc, antimony, tin, chromium and manganese) and mixed internal standard solution (indium, yttrium, holmium, and scandium) with the concentration of $10 \mathrm{mg} / \mathrm{L}$ were delivered by Perkin Elmer and certified for purity and concentration. A mixed working standard with the concentration of $1 \mathrm{mg} / \mathrm{L}$ was prepared by pipetting $5 \mathrm{~mL}$ of each stock solution into a $50 \mathrm{~mL}$ volumetric flask and diluted up to $50 \mathrm{~mL}$ with $0.2 \%$ nitric acid $\left(\mathrm{HNO}_{3}\right.$ Suprapur, Merck). This standard solution was used for calibration. All the laboratory apparatus were decontaminated by soaking in $10 \% \mathrm{v} / \mathrm{v} \mathrm{HNO}_{3}$ for 24 hours before analysis. Ultra-pure water with 18.2 M-ohm was used for the preparation of all solution and reagents.

\subsection{Digestion Procedure}

The dried fish samples were weighted for $0.5 \mathrm{~g}$ and digested with a mixture of 5 $\mathrm{mL}$ concentrated nitric acid and $2 \mathrm{~mL}$ of hydrogen peroxide in a microwave digestion system (Multiwave 3000, Anton Paar). The power profile for the digestion process was as follows: During the first phase, the power of the digestion system was set at $600 \mathrm{~W}$, followed by 5 minutes ramping and holding, respectively. At the second phase, the power was increased to $1400 \mathrm{~W}$ followed by 5 minutes ramping and 10 minutes holding time. Finally, in the third phase, the power was turned to zero withholding time of 15 minutes. After the digestion process, samples were filtered through a $0.45 \mu \mathrm{m}$ acid resistant membrane. The solution was transferred into a $25 \mathrm{ml}$ volumetric flask and diluted with ultrapure water. The analytical reagent blanks also prepared in the same manner but without the dried fish samples [6] [28].

\subsection{Heavy Metals Analysis}

The heavy metals were analyzed by Inductively Coupled Plasma Mass Spectrometry (ICP-MS) ELAN 9000 (Perkin Elmer, Sciex, Canada) equipped with a Meinhard concentric quartz nebuliser, cyclonic spray chamber, nickel sampler, nickel skimmer cones and an autosampler (Perkin Elmer). The calibration curves were established using a mixed working standard solution containing 1 $\mathrm{mg} / \mathrm{L}$ (Se, $\mathrm{Cd}, \mathrm{Pb}, \mathrm{Cu}, \mathrm{Zn}, \mathrm{Sb}, \mathrm{Sn}, \mathrm{Cr}$ and $\mathrm{Mn}$ ) as described before by subsequent dilution into concentrations of $10,40,70$ and $100 \mu \mathrm{g} / \mathrm{L}$ of each element, with 20 $\mu \mathrm{g} / \mathrm{L}$ of mixed internal standard (In, Y, Ho, and Sc). The calibration curve showed linearity with a correlation coefficient of higher than $0.9995\left(\mathrm{r}^{2}>\right.$ 0.9995). Limits of detection were determined for each element by using seven replicate aliquots of the fortified reagent blank $\left(0.2 \% \mathrm{HNO}_{3}+0.05 \mu \mathrm{g} / \mathrm{L}\right.$ of each element). Three times of the standard deviation of the aliquots readings were calculated to determine the detection limit and the sample analyses were conducted triplicate to ensure accuracy of the samples. The concentrations of heavy metals were converted to wet basis values in order to compare with PTWI. The formula as follows; wet weight concentration $=$ dry wet concentration $\times$ 
(100/100 moisture percentage) [6]. The moisture content of the fish was calculated based on the study by Tee et al., 1997 [29] and Nurnadia et al., 2011 [30].

\subsection{Quality Control}

The precision of the method was evaluated by adding $20 \mu \mathrm{g} / \mathrm{L}$ of the standard solution of multi-elements ( $\mathrm{Se}, \mathrm{Cd}, \mathrm{Pb}, \mathrm{Cu}, \mathrm{Zn}, \mathrm{Sb}, \mathrm{Sn}, \mathrm{Cr}$ and $\mathrm{Mn}$ ) to the fish sample. The concentration of the sample before and after the addition was measured. The recovery values of these elements were between acceptable ranges, which were within $85 \%$ to $115 \%$. The standard reference samples (NIST SRM ${ }^{\circ}$ 1946-Lake Superior Fish Tissue) were analyzed to ensure quality control of this analysis. The average recovery of reference standards was $90.7 \%$.

\subsection{Estimated Weekly Intake (EWI)}

To prevent human health impairment by heavy metals originating from dietary sources, the Joint FAO/WHO Expert Committee on Food Additives (JECFA) assigned a PTWI [31] for total $\mathrm{Cd}, \mathrm{Pb}, \mathrm{Cu}, \mathrm{Zn}$ and $\mathrm{Sn}$ that are 0.007, 0.025, 3.5, 7.0 and $14.0 \mathrm{mg} / \mathrm{kg}$ b.wt./week respectively [20] [21]. According to FAO 2009, fish consumption for an average age of Malaysian per capita is $160 \mathrm{~g} /$ day/person [32] [33]. The average body weight for the Malaysian population is $62 \mathrm{~kg}$ [34]. In order to estimate the risk of exposure from consuming fish, Estimated Weekly Intake (EWI) of elements of fish was calculated as follows [20]:

$$
\text { EWI }=\frac{\text { concentration of metals }(\mathrm{mg} / \mathrm{kg} \text {, wet weight }) \times \text { weekly consumption }}{\text { body weight }(\mathrm{kg})}
$$

\subsection{Health Risk Assessment Procedure}

Risk assessment is a tool to estimate the probability of health effects due to exposure to the hazard, which in this study is the exposure through consumption of fish. USEPA developed the oral reference dose (RfDs) for Se as $5 E^{-3}$ $\mathrm{mg} / \mathrm{kg} /$ day; $\mathrm{Cd}$ as $1 \mathrm{E}^{-3} \mathrm{mg} / \mathrm{kg} /$ day; $\mathrm{Zn}$ as $3 \mathrm{E}^{-1} \mathrm{mg} / \mathrm{kg} /$ day; $\mathrm{Sb}$ as $4 \mathrm{E}^{-4} \mathrm{mg} / \mathrm{kg} /$ day; and $\mathrm{Mn}$ as $1.4 \mathrm{E}^{-1} \mathrm{mg} / \mathrm{kg} /$ day (Risk Information System (IRIS), USEPA). There is no established reference of dose for $\mathrm{Pb}, \mathrm{Cu}, \mathrm{Sn}$ and $\mathrm{Cr}$ under IRIS USEPA. The health risks assessment for non-carcinogen metals were calculated based on the following equation [22] [35] [36]:

$$
\mathrm{HQ}=\frac{\mathrm{EF} \times \mathrm{ED} \times \mathrm{FIR} \times \mathrm{C}}{\mathrm{RfD} \times \mathrm{BW} \times \mathrm{AT}} \times 10^{-3}
$$

where HQ is chemical-specific Hazard Quotient; EF is the exposure frequency (350 days/year); ED is the exposure duration (30 years); FIR is the fish ingestion rate $(160 \mathrm{~g} /$ day/person); $\mathrm{C}$ is the metal concentration in the muscle of fishes ( $\mathrm{mg} / \mathrm{kg}$ wet weight); RfD is the oral reference dose (IRIS, USEPA); BW is the average adult body weight $(62 \mathrm{~kg})$ and $\mathrm{AT}$ is the average exposure time for non-carcinogen (365 days/year $\times$ number of exposure years, assuming 30 years).

A summation of the hazard quotients for all chemicals to which an individual 
is exposed was used to calculate the hazard index as described in following equation [35] [36]:

$$
\mathrm{HI}=\mathrm{HQ}_{\mathrm{Se}}+\mathrm{HQ}_{\mathrm{Cd}}+\mathrm{HQ}_{\mathrm{Zn}}+\mathrm{HQ}_{\mathrm{Sb}}+\mathrm{HQ}_{\mathrm{Mn}}
$$

where $\mathrm{HI}$ is the hazard index; $\mathrm{HQ}_{\mathrm{se}}$ is the target hazard quotient for Se intake; $\mathrm{HQ}_{\mathrm{Cd}}$ is the target hazard quotient for Cd intake; $\mathrm{HQ}_{\mathrm{Zn}}$ is the target hazard quotient for $\mathrm{Zn}$ intake; $\mathrm{HQ}_{\mathrm{sb}}$ is the target hazard quotient for $\mathrm{Sb}$ intake; $\mathrm{HQ}_{\mathrm{Mn}}$ is the target hazard quotient for $\mathrm{Mn}$ intake. If the $\mathrm{HQ}$ and $\mathrm{HI}$ values were greater than 1 , then it is implied that there is a potential non-carcinogenic health risk related to the studied metals.

\subsection{Data Analysis and Statistics}

Before statistical analysis, the data was cleaned and checked for discrepancies. The non-parametric technique was chosen for analysis as the data was not normally distributed because of the presence of outliers. The median was calculated using SPSS (version 11.5 for Windows, 2002, SPSS Inc.). The median was calculated from triplicate analysis of each sample and interquartile range was calculated to show dispersion of dataset. The statistical significance of difference was assessed using Mann-Whitney's (MW) test for two groups and Kruskal-Wallis's $(\mathrm{KW})$ test for three groups or more. The level of significance was designated as $\mathrm{p}$ $<0.05$ (5\%).

\section{Results}

\subsection{Heavy Metals Concentration in Marine Fish Samples}

A total of 296 marine fish samples were analyzed in this study. The samples included 177 samples of pelagic fish, which were classified into two families; $\mathrm{Ca}$ rangidae (80 samples) and Scrombidae (97 samples). There were 119 samples of demersal fish samples which were categorized into five families; Lutjanidae (24 samples), Latidae (14 samples), Dasyatidae (25 samples), Sciaenidae (25 samples), and Nemipteridae (31 samples). The size of fish from collected samples ranged between $12.0 \mathrm{~cm}$ (Selaroides leptolepis) to $142.0 \mathrm{~cm}$ (Himantura uarnak), the smallest weighed between $0.02 \mathrm{~kg}$ and $0.29 \mathrm{~kg}$ and the largest weighed between $2.26 \mathrm{~kg}$ and $3.04 \mathrm{~kg}$ (Table 1).

Heavy metals median concentration of nine elements ( $\mathrm{Se}, \mathrm{Cd}, \mathrm{Pb}, \mathrm{Cu}, \mathrm{Zn}, \mathrm{Sb}$, $\mathrm{Sn}, \mathrm{Cr}$ and $\mathrm{Mn}$ ) from marine fish samples were summarized in Table 2. Results were expressed in wet weight $(\mathrm{w} / \mathrm{w})$ basis for comparison later with PTWI. Overall, the heavy metals accumulation in marine fish samples was found to be dominated by $\mathrm{Zn}$ followed by $\mathrm{Sn}, \mathrm{Se}, \mathrm{Cu}, \mathrm{Mn}, \mathrm{Cr}, \mathrm{Pb}, \mathrm{Cd}$ and $\mathrm{Sb}$ which ranged between $15.9612 \mathrm{mg} / \mathrm{kg}(\mathrm{Zn})$ to $0.0002 \mathrm{mg} / \mathrm{kg}$ (Sb) wet weight. Among the heavy metals studied, $\mathrm{Zn}$ showed the highest level of accumulation and Decapterus macrosoma showed the highest concentration of $\mathrm{Zn}(15.9612 \mathrm{mg} / \mathrm{kg})$ whereas Otolithoides biauritus showed the lowest concentration of $\mathrm{Zn}(2.3000 \mathrm{mg} / \mathrm{kg})$. Sb showed the lowest level of accumulation, which was ranged between 0.0048 
$\mathrm{mg} / \mathrm{kg}$ (Decapterus macrosoma) and $0.0002 \mathrm{mg} / \mathrm{kg}$ (Selar crumenopthalmus, Otolithoides biauritus, Nemipterus tambuloides). Otolithoides biauritus demonstrated the lowest concentration for all heavy metal except for $\mathrm{Pb}$. The highest concentration of Cd $(0.0786 \mathrm{mg} / \mathrm{kg}), \mathrm{Zn}(15.9612 \mathrm{mg} / \mathrm{kg})$ and Sb $(0.0048 \mathrm{mg} / \mathrm{kg})$ were found to be in Decapterus macrosoma samples. Sample Himantura gerrardi demonstrated the highest concentration of $\mathrm{Pb}(0.057 \mathrm{mg} / \mathrm{kg})$ while sample Nemipterus tambuloides showed the highest concentration of Sn (5.717 $\mathrm{mg} / \mathrm{kg}$ ). The highest concentration of Se was found in Thunnus tonggol (1.221 $\mathrm{mg} / \mathrm{kg}$ ) while the highest concentration of $\mathrm{Cu}$ was found in Megalaspis cordyla $(1.614 \mathrm{mg} / \mathrm{kg})$. Nemipterus nemurus showed the highest concentration of $\mathrm{Cr}$ $(0.411 \mathrm{mg} / \mathrm{kg})$ while Dasyatis zugei showed the highest concentration of $\mathrm{Mn}$ $(1.542 \mathrm{mg} / \mathrm{kg})$.

Table 1. List of marine fish samples collected from LKIM complexes and wholesale market in Peninsular Malaysia.

\begin{tabular}{|c|c|c|c|c|c|}
\hline No. & Groups/family/species & Common name & $\mathbf{n}$ & Size range $(\mathrm{cm})$ & Weight range (kg) \\
\hline & Pelagic fish & & & & \\
\hline & Carangidae (80) & & & & \\
\hline 1. & Selaroides leptolepis & Yellowstripe scad & 13 & $12.6-19.6$ & $0.028-0.290$ \\
\hline 2. & Selar boops & Oxeye scad & 3 & $19.0-24.8$ & $0.009-0.212$ \\
\hline 3. & Selar crumenopthalmus & Bigeye scad & 1 & 23.8 & 0.186 \\
\hline 4. & Atule mate & Yellowtail scad & 4 & $23.1-26.3$ & $0.150-0.224$ \\
\hline 5. & Caranx sexfasciatus & Bigeye trevally & 1 & 22.7 & 0.140 \\
\hline 6. & Seriola dumerili & Greater amberjack & 1 & 19.6 & 0.106 \\
\hline 7. & Decapterus kurroides & Redtail scad & 4 & $18.5-26.8$ & $0.066-0.224$ \\
\hline 8. & Decapterus muruadsi & Round scad & 7 & $18.1-36.2$ & $0.071-0.294$ \\
\hline 9. & Decapterus russelli & Slender scad & 10 & $16.5-30.1$ & $0.052-0.420$ \\
\hline 10. & Decapterus macrosoma & Shortfin scad & 1 & - & - \\
\hline 11. & Megalaspis cordyla & Torpedo scad & 20 & $22.2-34.3$ & $0.101-0.300$ \\
\hline \multirow[t]{3}{*}{12.} & Parastromateus niger & Black pomfret & 15 & $15.6-40.3$ & $0.082-1.466$ \\
\hline & & Total & 80 & & \\
\hline & Scrombidae (97) & & & & \\
\hline 13. & Rastrelliger kanagurta & Indian mackerel & 13 & $13.4-24.3$ & $0.200-0.360$ \\
\hline 14. & Rastrelliger faughni & Faughn's mackerel & 6 & $19.0-21.8$ & $0.100-0.180$ \\
\hline 15. & Rastrelliger brachysoma & Indo-Pacific mackerel & 3 & $16.6-25.5$ & $0.050-0.280$ \\
\hline 16. & Scomber australasicus & Blue mackerel & 18 & $17.0-22.4$ & $0.040-0.152$ \\
\hline 17. & Scomberomorus guttatus & Indo-Pacific king mackerel & 12 & $29.6-55.5$ & $0.176-1.066$ \\
\hline 18. & Scomberomorus commerson & $\begin{array}{c}\text { Narrowbarred } \\
\text { Spanish mackerel }\end{array}$ & 14 & $40.1-85.5$ & $0.394-4.550$ \\
\hline 19. & Gymnosarda unicolor & Dogtooth tuna & 10 & $19.8-49.5$ & $0.082-5.000$ \\
\hline 20. & Sarda orientalis & Striped bonito & 6 & $25.5-48.0$ & $0.232-1.600$ \\
\hline
\end{tabular}




\section{Continued}

\begin{tabular}{|c|c|c|c|c|c|}
\hline 21. & Thunnus tonggol & Longtail tuna & 8 & $17.2-50.0$ & $0.082-1.733$ \\
\hline 22. & Auxis thazard thazard & Frigate tuna & 2 & $29.6-31.2$ & $0.334-0.412$ \\
\hline \multirow[t]{4}{*}{23.} & Euthymus affinis & Kawakawa & 5 & $29.4-69.3$ & $0.370-4.733$ \\
\hline & & Total & 97 & & \\
\hline & Demersal fish & & & & \\
\hline & Lutjanidae (24) & & & & \\
\hline 24. & Lutjanus argentimaculatus & Mangrove red snapper & 3 & $38.3-38.5$ & $1.033-1.175$ \\
\hline 25. & Lutjanus gibbus & Humpback red snapper & 1 & 20.2 & 0.148 \\
\hline 26. & Lutjanus sebae & Emperor red snapper & 11 & $18.1-62.0$ & $0.102-3.300$ \\
\hline 27. & Lutjanus malabaricus & Malabar blood snapper & 5 & $27.9-49.75$ & $0.394-1.175$ \\
\hline \multirow[t]{3}{*}{28.} & Lutjanus russellii & John's snapper & 4 & 66.0 & 4.050 \\
\hline & & Total & 24 & & \\
\hline & Latidae (15) & & & & \\
\hline 29. & Lates calcarifer & Giant sea perch & 11 & $26.6-71.6$ & $0.046-4.650$ \\
\hline \multirow[t]{3}{*}{30.} & Psammoperca waigiensis & Waigeu sea perch & 4 & $34.6-38.3$ & $0.396-0.875$ \\
\hline & & Total & 15 & & \\
\hline & Dasyatidae (25) & & & & \\
\hline 31. & Himantura gerrardi & Sharpnose stingray & 10 & $21.4-116.3$ & $0.208-2.880$ \\
\hline 32. & Neotrygon kuhlii & Bluespotted stingray & 7 & $45.7-114.0$ & $0.510-4.300$ \\
\hline 33. & Dasyatis zugei & Pale-edged stingray & 5 & $40.7-147.3$ & $0.188-1.157$ \\
\hline \multirow[t]{3}{*}{34.} & Himantura uarnak & Honeycomb stingray & 3 & $131.6-142.0$ & $2.266-3.040$ \\
\hline & & Total & 25 & & \\
\hline & Sciaenidae (25) & & & & \\
\hline 35. & Chrysochir aureus & Reeve's croaker & 3 & $19.0-25.4$ & $0.074-0.220$ \\
\hline 36. & Otolithoides ruber & Tigertooth croaker & 6 & $13.0-24.2$ & $0.04-0.113$ \\
\hline 37. & Nibea soldado & Soldier croaker & 15 & $15.3-21.6$ & $0.041-0.274$ \\
\hline \multirow[t]{3}{*}{38.} & Otolithoides biauritus & Bronze croaker & 1 & 20.9 & 0.132 \\
\hline & & Total & 25 & & \\
\hline & Nemipteridae (31) & & & & \\
\hline 39. & Nemipterus bathybius & Yellowbelly threadfin bream & 6 & $15.7-33.7$ & $0.063-0.516$ \\
\hline 40. & Nemipterus japonicus & Japanese threadfin bream & 11 & $16.9-29.2$ & $0.063-0.212$ \\
\hline 41. & Nemipterus furcosus & Forktail threadfin bream & 3 & $18.2-21.4$ & $0.102-0.162$ \\
\hline 42. & Nemipterus thosaporni & Threadfin bream & 4 & $17.0-24.0$ & $0.059-0.210$ \\
\hline 43. & Nemipterus tambuloides & Fivelined threadfin bream & 2 & $17.5-21.4$ & $0.086-0.109$ \\
\hline 44. & Nemipterus nematophorus & Doublewhip threadfin bream & 2 & $16.1-25.6$ & $0.058-0.154$ \\
\hline 45. & Nemipterus marginatus & Red filament threadfin bream & 2 & $23.0-25.5$ & $0.11-0.24$ \\
\hline \multirow[t]{2}{*}{46.} & Nemipterus nemurus & Redspine threadfin bream & 1 & 17.8 & 0.096 \\
\hline & & Total & 31 & & \\
\hline
\end{tabular}


Table 2. Concentration of heavy metals in marine fish sampled from LKIM complexes and wholesale market in Peninsular Malaysia.

\begin{tabular}{|c|c|c|c|c|c|c|c|c|c|c|c|c|}
\hline \multirow[t]{2}{*}{ No. } & \multirow[t]{2}{*}{ Groups/family/species } & \multirow[t]{2}{*}{ Common name } & \multirow[t]{2}{*}{$\mathbf{n}$} & \multicolumn{9}{|c|}{ Concentration of heavy metals in median $\mathrm{mg} / \mathrm{kg}$, wet weight (IQR) } \\
\hline & & & & $\mathrm{Se}$ & $\mathrm{Cd}$ & $\mathrm{Pb}$ & $\mathrm{Cu}$ & $\mathrm{Zn}$ & $\mathrm{Sb}$ & Sn & $\mathrm{Cr}$ & Mn \\
\hline \multicolumn{13}{|c|}{ Pelagic fish } \\
\hline \multicolumn{13}{|c|}{ Carangidae (80) } \\
\hline 1 & Selaroides leptolepis & Yellowstripe scad & 13 & $\begin{array}{c}0.438 \\
(0.1)\end{array}$ & $\begin{array}{c}0.019 \\
(0.0)\end{array}$ & $\begin{array}{c}0.014 \\
(0.1)\end{array}$ & $\begin{array}{c}0.500 \\
(0.9)\end{array}$ & $\begin{array}{c}7.201 \\
(1.5)\end{array}$ & $\begin{array}{c}0.001 \\
(0.0)\end{array}$ & $\begin{array}{l}1.789 \\
(3.3)\end{array}$ & $\begin{array}{c}0.216 \\
(0.6)\end{array}$ & $\begin{array}{c}0.158 \\
(0.1)\end{array}$ \\
\hline 2 & Selar boops & Oxeye scad & 3 & $\begin{array}{c}0.389 \\
(0.0)\end{array}$ & $\begin{array}{c}0.008 \\
(0.0)\end{array}$ & $\begin{array}{c}0.015 \\
(0.0)\end{array}$ & $\begin{array}{c}0.646 \\
(0.0)\end{array}$ & $\begin{array}{c}5.373 \\
(0.0)\end{array}$ & $\begin{array}{c}0.001 \\
(0.0)\end{array}$ & $\begin{array}{c}1.419 \\
(0.0)\end{array}$ & $\begin{array}{c}0.347 \\
(0.0)\end{array}$ & $\begin{array}{c}0.158 \\
(0.0)\end{array}$ \\
\hline 3 & Selar crumenopthalmus & Bigeye scad & 1 & $\begin{array}{c}0.738 \\
(0.0)\end{array}$ & $\begin{array}{c}0.029 \\
(0.0)\end{array}$ & $\begin{array}{c}0.017 \\
(0.0)\end{array}$ & $\begin{array}{c}0.426 \\
(0.0)\end{array}$ & $\begin{array}{c}5.743 \\
(0.0)\end{array}$ & $\begin{array}{c}0.000 \\
(0.0)\end{array}$ & $\begin{array}{c}3.860 \\
(0.0)\end{array}$ & $\begin{array}{c}0.114 \\
(0.0)\end{array}$ & $\begin{array}{c}0.229 \\
(0.0)\end{array}$ \\
\hline 4 & Atule mate & Yellowtail scad & 4 & $\begin{array}{l}0.587 \\
(0.11)\end{array}$ & $\begin{array}{c}0.007 \\
(0.0)\end{array}$ & $\begin{array}{c}0.002 \\
(0.0)\end{array}$ & $\begin{array}{c}0.924 \\
(0.0)\end{array}$ & $\begin{array}{l}8.487 \\
(4.0)\end{array}$ & $\begin{array}{c}0.000 \\
(0.0)\end{array}$ & $\begin{array}{c}1.260 \\
(0.0)\end{array}$ & $\begin{array}{c}0.264 \\
(0.0)\end{array}$ & $\begin{array}{c}0.275 \\
(0.0)\end{array}$ \\
\hline 5 & Caranx sexfasciatus & Bigeye trevally & 1 & $\begin{array}{c}0.598 \\
(0.0)\end{array}$ & $\begin{array}{c}0.013 \\
(0.0)\end{array}$ & $\begin{array}{c}0.019 \\
(0.0)\end{array}$ & $\begin{array}{c}0.698 \\
(0.0)\end{array}$ & $\begin{array}{c}9.491 \\
(0.0)\end{array}$ & $\begin{array}{c}0.001 \\
(0.0)\end{array}$ & $\begin{array}{c}2.282 \\
(0.0)\end{array}$ & $\begin{array}{c}0.335 \\
(0.0)\end{array}$ & $\begin{array}{c}0.198 \\
(0.0)\end{array}$ \\
\hline 6 & Seriola dumerili & Greater amberjack & 1 & $\begin{array}{c}0.376 \\
(0.0)\end{array}$ & $\begin{array}{c}0.025 \\
(0.0)\end{array}$ & $\begin{array}{c}0.049 \\
(0.0)\end{array}$ & $\begin{array}{c}0.764 \\
(0.0)\end{array}$ & $\begin{array}{c}5.299 \\
(0.0)\end{array}$ & $\begin{array}{c}0.000 \\
(0.0)\end{array}$ & $\begin{array}{c}2.971 \\
(0.0)\end{array}$ & $\begin{array}{c}0.452 \\
(0.0)\end{array}$ & $\begin{array}{c}0.255 \\
(0.0)\end{array}$ \\
\hline 7 & Decapterus kurroides & Redtail scad & 4 & $\begin{array}{l}0.756 \\
(0.49)\end{array}$ & $\begin{array}{c}0.038 \\
(0.1)\end{array}$ & $\begin{array}{c}0.009 \\
(0.0)\end{array}$ & $\begin{array}{c}0.873 \\
(0.1)\end{array}$ & $\begin{array}{c}8.505 \\
(5.5)\end{array}$ & $\begin{array}{c}0.001 \\
(0.0)\end{array}$ & $\begin{array}{c}1.614 \\
(5.0)\end{array}$ & $\begin{array}{c}0.237 \\
(0.2)\end{array}$ & $\begin{array}{c}0.216 \\
(0.3)\end{array}$ \\
\hline 8 & Decapterus muruadsi & Round scad & 7 & $\begin{array}{l}0.822 \\
(0.18)\end{array}$ & $\begin{array}{c}0.023 \\
(0.1)\end{array}$ & $\begin{array}{c}0.012 \\
(0.1)\end{array}$ & $\begin{array}{c}0.892 \\
(1.1)\end{array}$ & $\begin{array}{c}8.109 \\
(3.1)\end{array}$ & $\begin{array}{c}0.000 \\
(0.0)\end{array}$ & $\begin{array}{c}1.932 \\
(3.8)\end{array}$ & $\begin{array}{c}0.148 \\
(0.2)\end{array}$ & $\begin{array}{c}0.181 \\
(0.1)\end{array}$ \\
\hline 9 & Decapterus russelli & Slender scad & 10 & $\begin{array}{l}0.998 \\
(0.39)\end{array}$ & $\begin{array}{c}0.027 \\
(0.0)\end{array}$ & $\begin{array}{c}0.012 \\
(0.0)\end{array}$ & $\begin{array}{l}1.328 \\
(0.4)\end{array}$ & $\begin{array}{c}7.352 \\
(3.0)\end{array}$ & $\begin{array}{c}0.001 \\
(0.0)\end{array}$ & $\begin{array}{c}2.260 \\
(2.9)\end{array}$ & $\begin{array}{c}0.222 \\
(0.2)\end{array}$ & $\begin{array}{c}0.223 \\
(0.1)\end{array}$ \\
\hline 10 & Decapterus macrosoma & Shortfin scad & 1 & $\begin{array}{l}1.075 \\
(0.0)\end{array}$ & $\begin{array}{c}0.079 \\
(0.0)\end{array}$ & $\begin{array}{c}0.017 \\
(0.0)\end{array}$ & $\begin{array}{c}0.877 \\
(0.0)\end{array}$ & $\begin{array}{c}15.961 \\
(0.0)\end{array}$ & $\begin{array}{c}0.005 \\
(0.0)\end{array}$ & $\begin{array}{l}1.317 \\
(0.0)\end{array}$ & $\begin{array}{c}0.258 \\
(0.0)\end{array}$ & $\begin{array}{c}0.273 \\
(0.0)\end{array}$ \\
\hline 11 & Megalaspis cordyla & Torpedo scad & 20 & $\begin{array}{c}0.667 \\
(0.2)\end{array}$ & $\begin{array}{c}0.023 \\
(0.0)\end{array}$ & $\begin{array}{c}0.012 \\
(0.0)\end{array}$ & $\begin{array}{l}1.614 \\
(1.6)\end{array}$ & $\begin{array}{c}7.008 \\
(3.1)\end{array}$ & $\begin{array}{c}0.001 \\
(0.0)\end{array}$ & $\begin{array}{c}2.022 \\
(1.9)\end{array}$ & $\begin{array}{c}0.270 \\
(0.1)\end{array}$ & $\begin{array}{c}0.250 \\
(0.2)\end{array}$ \\
\hline \multirow[t]{3}{*}{12} & Parastromateus niger & Black pomfret & 15 & $\begin{array}{c}0.573 \\
(0.2)\end{array}$ & $\begin{array}{c}0.015 \\
(0.0)\end{array}$ & $\begin{array}{c}0.035 \\
(0.0)\end{array}$ & $\begin{array}{c}0.349 \\
(0.5)\end{array}$ & $\begin{array}{c}5.124 \\
(2.1)\end{array}$ & $\begin{array}{c}0.001 \\
(0.0)\end{array}$ & $\begin{array}{l}1.914 \\
(2.7)\end{array}$ & $\begin{array}{c}0.230 \\
(0.2)\end{array}$ & $\begin{array}{c}0.385 \\
(0.4)\end{array}$ \\
\hline & & Total & 80 & 0.668 & 0.025 & 0.018 & 0.824 & 7.804 & 0.001 & 2.053 & 0.258 & 0.233 \\
\hline & \multicolumn{12}{|l|}{ Scrombidae (97) } \\
\hline 13 & Rastrelliger kanagurta & Indian mackerel & 13 & $\begin{array}{c}0.793 \\
(0.2)\end{array}$ & $\begin{array}{c}0.014 \\
(0.0)\end{array}$ & $\begin{array}{c}0.033 \\
(0.1)\end{array}$ & $\begin{array}{c}0.971 \\
(0.4)\end{array}$ & $\begin{array}{c}9.082 \\
(5.6)\end{array}$ & $\begin{array}{c}0.001 \\
(0.0)\end{array}$ & $\begin{array}{c}2.465 \\
(3.1)\end{array}$ & $\begin{array}{c}0.312 \\
(0.4)\end{array}$ & $\begin{array}{c}0.222 \\
(0.2)\end{array}$ \\
\hline 14 & Rastrelliger faughni & Faughn's mackerel & 6 & $\begin{array}{c}0.832 \\
(0.3)\end{array}$ & $\begin{array}{c}0.030 \\
(0.1)\end{array}$ & $\begin{array}{c}0.041 \\
(0.1)\end{array}$ & $\begin{array}{l}1.281 \\
(3.1)\end{array}$ & $\begin{array}{c}9.531 \\
(3.9)\end{array}$ & $\begin{array}{c}0.000 \\
(0.0)\end{array}$ & $\begin{array}{l}1.608 \\
(3.4)\end{array}$ & $\begin{array}{c}0.297 \\
(0.4)\end{array}$ & $\begin{array}{c}0.428 \\
(1.0)\end{array}$ \\
\hline 15 & Rastrelliger brachysoma & Indo-Pacific mackerel & 3 & $\begin{array}{c}0.726 \\
(0.0)\end{array}$ & $\begin{array}{c}0.010 \\
(0.0)\end{array}$ & $\begin{array}{c}0.034 \\
(0.0)\end{array}$ & $\begin{array}{c}0.908 \\
(0.0)\end{array}$ & $\begin{array}{c}9.554 \\
(0.0)\end{array}$ & $\begin{array}{c}0.001 \\
(0.0)\end{array}$ & $\begin{array}{l}1.367 \\
(0.0)\end{array}$ & $\begin{array}{c}0.165 \\
(0.0)\end{array}$ & $\begin{array}{r}0.177 \\
(0.0)\end{array}$ \\
\hline 16 & Scomber australasicus & Blue mackerel & 18 & $\begin{array}{c}0.895 \\
(0.3)\end{array}$ & $\begin{array}{c}0.021 \\
(0.0)\end{array}$ & $\begin{array}{c}0.021 \\
(0.0)\end{array}$ & $\begin{array}{c}0.943 \\
(0.7)\end{array}$ & $\begin{array}{c}6.500 \\
(3.1)\end{array}$ & $\begin{array}{c}0.001 \\
(0.0)\end{array}$ & $\begin{array}{c}2.122 \\
(2.6)\end{array}$ & $\begin{array}{c}0.179 \\
(0.1)\end{array}$ & $\begin{array}{c}0.204 \\
(0.2)\end{array}$ \\
\hline 17 & $\begin{array}{l}\text { Scomberomorus } \\
\text { guttatus }\end{array}$ & $\begin{array}{l}\text { Indo-Pacific } \\
\text { king mackerel }\end{array}$ & 12 & $\begin{array}{c}0.384 \\
(0.1)\end{array}$ & $\begin{array}{c}0.003 \\
(0.0)\end{array}$ & $\begin{array}{c}0.011 \\
(0.0)\end{array}$ & $\begin{array}{c}0.330 \\
(0.1)\end{array}$ & $\begin{array}{l}4.253 \\
(1.0)\end{array}$ & $\begin{array}{c}0.001 \\
(0.0)\end{array}$ & $\begin{array}{c}2.666 \\
(3.5)\end{array}$ & $\begin{array}{c}0.143 \\
(0.1)\end{array}$ & $\begin{array}{r}0.095 \\
(0.1)\end{array}$ \\
\hline 18 & $\begin{array}{c}\text { Scomberomorus } \\
\text { commerson }\end{array}$ & $\begin{array}{c}\text { Narrowbarred Spanish } \\
\text { mackerel }\end{array}$ & 14 & $\begin{array}{l}0.530 \\
(0.2)\end{array}$ & $\begin{array}{l}0.006 \\
(0.0)\end{array}$ & $\begin{array}{l}0.017 \\
(0.0)\end{array}$ & $\begin{array}{l}0.301 \\
(0.5)\end{array}$ & $\begin{array}{c}4.69292 . \\
3)\end{array}$ & $\begin{array}{c}0.001 \\
(0.0)\end{array}$ & $\begin{array}{l}1.358 \\
(2.0)\end{array}$ & $\begin{array}{c}0.256 \\
(0.2)\end{array}$ & $\begin{array}{l}0.112 \\
(0.2)\end{array}$ \\
\hline
\end{tabular}




\section{Continued}

\begin{tabular}{|c|c|c|c|c|c|c|c|c|c|c|c|c|}
\hline 19 & Gymnosarda unicolor & Dogtooth tuna & 10 & $\begin{array}{l}0.994 \\
(0.66)\end{array}$ & $\begin{array}{c}0.014 \\
(0.0)\end{array}$ & $\begin{array}{c}0.020 \\
(0.0)\end{array}$ & $\begin{array}{c}0.936 \\
(1.6)\end{array}$ & $\begin{array}{c}7.530 \\
(9.3)\end{array}$ & $\begin{array}{c}0.001 \\
(0.0)\end{array}$ & $\begin{array}{c}1.814 \\
(1.1)\end{array}$ & $\begin{array}{c}0.218 \\
(0.1)\end{array}$ & $\begin{array}{c}0.203 \\
(0.1)\end{array}$ \\
\hline 20 & Sarda orientalis & Striped bonito & 6 & $\begin{array}{c}0.861 \\
(0.4)\end{array}$ & $\begin{array}{c}0.014 \\
(0.0)\end{array}$ & $\begin{array}{c}0.014 \\
(0.0)\end{array}$ & $\begin{array}{c}1.084 \\
(0.8)\end{array}$ & $\begin{array}{c}7.790 \\
(5.8)\end{array}$ & $\begin{array}{c}0.001 \\
(0.0)\end{array}$ & $\begin{array}{c}2.174 \\
(1.8)\end{array}$ & $\begin{array}{c}0.236 \\
(0.3)\end{array}$ & $\begin{array}{c}0.182 \\
(0.2)\end{array}$ \\
\hline 21 & Thunnus tonggol & Longtail tuna & 8 & $\begin{array}{l}1.221 \\
(0.3)\end{array}$ & $\begin{array}{c}0.008 \\
(0.0)\end{array}$ & $\begin{array}{c}0.012 \\
(0.0)\end{array}$ & $\begin{array}{c}1.530 \\
(0.7)\end{array}$ & $\begin{array}{c}7.784 \\
(5.0)\end{array}$ & $\begin{array}{c}0.001 \\
(0.0)\end{array}$ & $\begin{array}{c}1.629 \\
(2.4)\end{array}$ & $\begin{array}{c}0.197 \\
(0.2)\end{array}$ & $\begin{array}{c}0.194 \\
(0.1)\end{array}$ \\
\hline 22 & Auxis thazard thazard & Frigate tuna & 2 & $\begin{array}{c}0.931 \\
(0.0)\end{array}$ & $\begin{array}{c}0.012 \\
(0.0)\end{array}$ & $\begin{array}{c}0.007 \\
(0.0)\end{array}$ & $\begin{array}{c}1.046 \\
(0.0)\end{array}$ & $\begin{array}{c}6.944 \\
(0.0)\end{array}$ & $\begin{array}{c}0.000 \\
(0.0)\end{array}$ & $\begin{array}{c}0.986 \\
(0.0)\end{array}$ & $\begin{array}{c}0.147 \\
(0.0)\end{array}$ & $\begin{array}{c}0.125 \\
(0.0)\end{array}$ \\
\hline \multirow[t]{3}{*}{23} & Euthymus affinis & Kawakawa & 5 & $\begin{array}{l}0.779 \\
(0.52)\end{array}$ & $\begin{array}{c}0.007 \\
(0.0)\end{array}$ & $\begin{array}{c}0.021 \\
(0.0)\end{array}$ & $\begin{array}{c}0.941 \\
(0.0)\end{array}$ & $\begin{array}{c}9.550 \\
(6.1)\end{array}$ & $\begin{array}{c}0.001 \\
(0.0)\end{array}$ & $\begin{array}{l}1.325 \\
(0.0)\end{array}$ & $\begin{array}{c}0.300 \\
(0.0)\end{array}$ & $\begin{array}{r}0.160 \\
(0.0)\end{array}$ \\
\hline & & Total & 97 & 0.813 & 0.013 & 0.021 & 0.934 & 7.564 & 0.001 & 1.774 & 0.223 & 0.191 \\
\hline & & Total (pelagic fish) & 177 & 0.738 & 0.019 & 0.019 & 0.877 & 7.690 & 0.001 & 1.920 & 0.241 & 0.213 \\
\hline
\end{tabular}

\section{Demersal fish}

\section{Lutjanidae (24)}

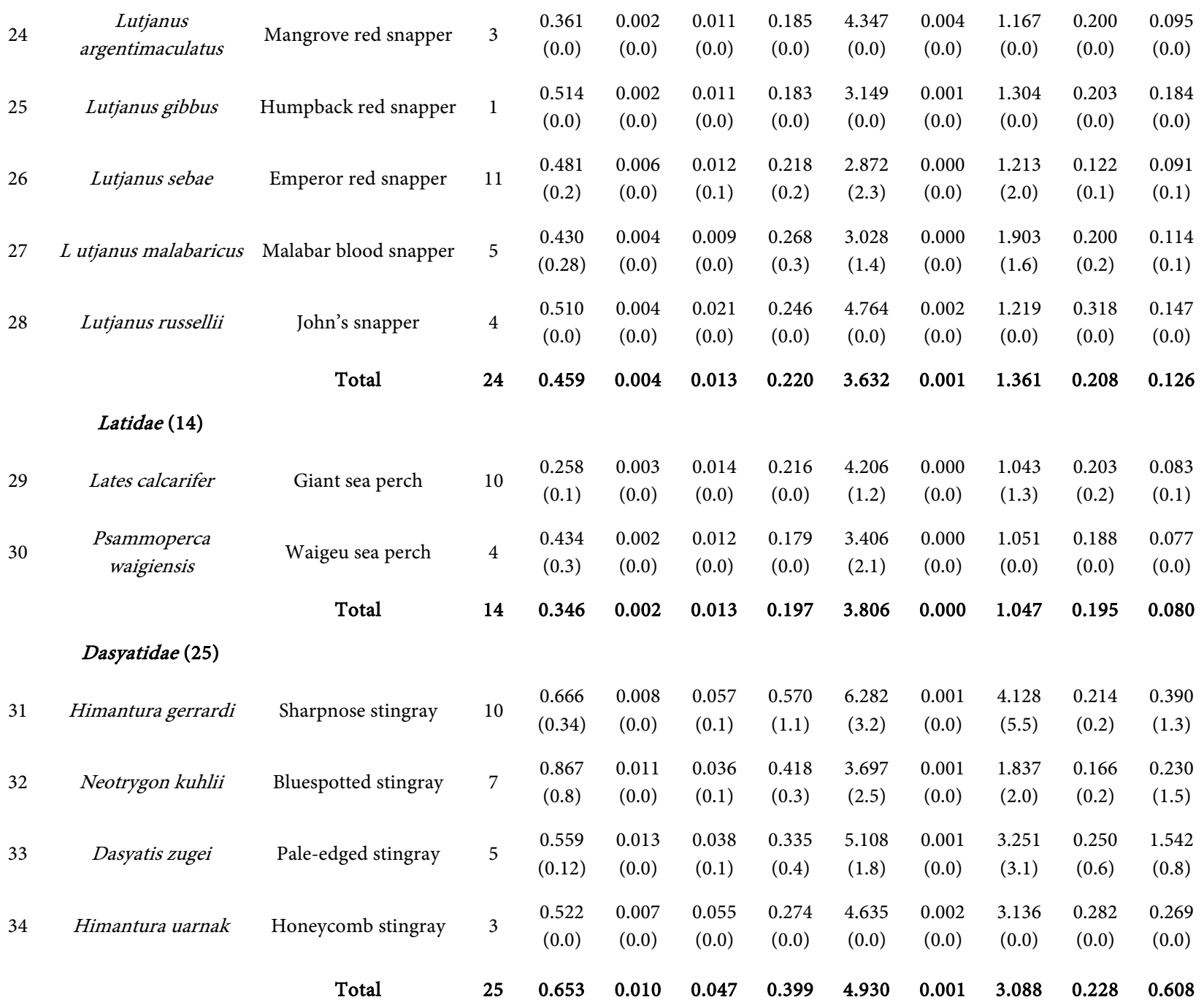

Sciaenidae (25) 


\section{Continued}

\begin{tabular}{|c|c|c|c|c|c|c|c|c|c|c|c|c|}
\hline 35 & Chrysochir aureus & Reeve's croaker & 3 & $\begin{array}{c}0.469 \\
(0.0)\end{array}$ & $\begin{array}{l}0.002 \\
(0.0)\end{array}$ & $\begin{array}{c}0.011 \\
(0.0)\end{array}$ & $\begin{array}{c}0.218 \\
(0.0)\end{array}$ & $\begin{array}{r}4.002 \\
(0.0)\end{array}$ & $\begin{array}{c}0.001 \\
(0.0)\end{array}$ & $\begin{array}{r}2.062 \\
(0.0)\end{array}$ & $\begin{array}{c}0.230 \\
(0.0)\end{array}$ & $\begin{array}{r}0.140 \\
(0.0)\end{array}$ \\
\hline 36 & Otolithoides ruber & Tigertooth croaker & 6 & $\begin{array}{c}0.492 \\
(0.1)\end{array}$ & $\begin{array}{c}0.004 \\
(0.0)\end{array}$ & $\begin{array}{c}0.016 \\
(0.0)\end{array}$ & $\begin{array}{c}0.290 \\
(0.3)\end{array}$ & $\begin{array}{l}4.341 \\
(3.8)\end{array}$ & $\begin{array}{c}0.001 \\
(0.0)\end{array}$ & $\begin{array}{l}3.465 \\
(3.6)\end{array}$ & $\begin{array}{c}0.220 \\
(0.5)\end{array}$ & $\begin{array}{r}0.296 \\
(0.3)\end{array}$ \\
\hline 37 & Nibea soldado & Soldier croaker & 15 & $\begin{array}{c}0.559 \\
(0.1)\end{array}$ & $\begin{array}{l}0.005 \\
(0.0)\end{array}$ & $\begin{array}{c}0.020 \\
(0.0)\end{array}$ & $\begin{array}{r}0.333 \\
(0.2)\end{array}$ & $\begin{array}{l}4.462 \\
(1.0)\end{array}$ & $\begin{array}{c}0.001 \\
(0.0)\end{array}$ & $\begin{array}{r}3.239 \\
(2.1)\end{array}$ & $\begin{array}{r}0.210 \\
(0.2)\end{array}$ & $\begin{array}{r}0.237 \\
(0.3)\end{array}$ \\
\hline \multirow[t]{3}{*}{38} & Otolithoides biauritus & Bronze croaker & 1 & $\begin{array}{l}0.074 \\
(0.0)\end{array}$ & $\begin{array}{l}0.001 \\
(0.0)\end{array}$ & $\begin{array}{c}0.005 \\
(0.0)\end{array}$ & $\begin{array}{c}0.039 \\
(0.0)\end{array}$ & $\begin{array}{r}2.300 \\
(0.0)\end{array}$ & $\begin{array}{c}0.000 \\
(0.0)\end{array}$ & $\begin{array}{c}0.283 \\
(0.0)\end{array}$ & $\begin{array}{c}0.016 \\
(0.0)\end{array}$ & $\begin{aligned} 0.065 \\
(0.0)\end{aligned}$ \\
\hline & & Total & 25 & 0.398 & 0.003 & 0.013 & 0.220 & 3.776 & 0.001 & 2.262 & 0.169 & 0.184 \\
\hline & \multicolumn{12}{|l|}{ Nemipteridae (31) } \\
\hline 39 & Nemipterus bathybius & $\begin{array}{c}\text { Yellowbelly threadfin } \\
\text { bream }\end{array}$ & 6 & $\begin{array}{c}0.544 \\
(0.4)\end{array}$ & $\begin{array}{c}0.006 \\
(0.0)\end{array}$ & $\begin{array}{c}0.011 \\
(0.0)\end{array}$ & $\begin{array}{c}0.249 \\
(0.1)\end{array}$ & $\begin{array}{r}2.875 \\
(1.2)\end{array}$ & $\begin{array}{c}0.001 \\
(0.0)\end{array}$ & $\begin{array}{c}3.256 \\
(2.1)\end{array}$ & $\begin{array}{c}0.200 \\
(0.1)\end{array}$ & $\begin{array}{r}0.208 \\
(0.1)\end{array}$ \\
\hline 40 & Nemipterus japonicus & $\begin{array}{c}\text { Japanese threadfin } \\
\text { bream }\end{array}$ & 11 & $\begin{array}{c}0.512 \\
(0.1)\end{array}$ & $\begin{array}{c}0.016 \\
(0.0)\end{array}$ & $\begin{array}{c}0.021 \\
(0.0)\end{array}$ & $\begin{array}{c}0.261 \\
(0.4)\end{array}$ & $\begin{array}{c}2.866 \\
(1.7)\end{array}$ & $\begin{array}{c}0.001 \\
(0.0)\end{array}$ & $\begin{array}{r}3.485 \\
(2.0)\end{array}$ & $\begin{array}{l}0.277 \\
(0.7)\end{array}$ & $\begin{array}{l}0.381 \\
(0.3)\end{array}$ \\
\hline 41 & Nemipterus furcosus & Forktail threadfin bream & 3 & $\begin{array}{c}0.543 \\
(0.0)\end{array}$ & $\begin{array}{c}0.004 \\
(0.0)\end{array}$ & $\begin{array}{c}0.013 \\
(0.0)\end{array}$ & $\begin{array}{r}0.281 \\
(0.0)\end{array}$ & $\begin{array}{r}3.143 \\
(0.0)\end{array}$ & $\begin{array}{c}0.000 \\
(0.0)\end{array}$ & $\begin{array}{l}1.554 \\
(0.0)\end{array}$ & $\begin{array}{c}0.228 \\
(0.0)\end{array}$ & $\begin{array}{r}0.217 \\
(0.0)\end{array}$ \\
\hline 42 & Nemipterus thosaporni & Threadfin bream & 4 & $\begin{array}{c}0.478 \\
(0.0)\end{array}$ & $\begin{array}{c}0.023 \\
(0.0)\end{array}$ & $\begin{array}{c}0.028 \\
(0.0)\end{array}$ & $\begin{array}{c}0.256 \\
(0.0)\end{array}$ & $\begin{array}{c}3.516 \\
(0.7)\end{array}$ & $\begin{array}{c}0.000 \\
(0.0)\end{array}$ & $\begin{array}{r}2.251 \\
(0.0)\end{array}$ & $\begin{array}{c}0.167 \\
(0.0)\end{array}$ & $\begin{array}{r}0.290 \\
(0.0)\end{array}$ \\
\hline 43 & $\begin{array}{l}\text { Nemipterus } \\
\text { tambuloides }\end{array}$ & $\begin{array}{c}\text { Fivelined threadfin } \\
\text { bream }\end{array}$ & 2 & $\begin{array}{c}0.575 \\
(0.0)\end{array}$ & $\begin{array}{c}0.008 \\
(0.0)\end{array}$ & ND & $\begin{array}{c}0.188 \\
(0.0)\end{array}$ & $\begin{array}{r}3.933 \\
(0.0)\end{array}$ & $\begin{array}{c}0.000 \\
(0.0)\end{array}$ & $\begin{array}{r}5.717 \\
(0.0)\end{array}$ & $\begin{array}{c}0.132 \\
(0.0)\end{array}$ & $\begin{array}{r}0.280 \\
(0.0)\end{array}$ \\
\hline 44 & $\begin{array}{c}\text { Nemipterus } \\
\text { nematophorus }\end{array}$ & $\begin{array}{l}\text { Doublewhip threadfin } \\
\text { bream }\end{array}$ & 2 & $\begin{array}{c}0.543 \\
(0.0)\end{array}$ & $\begin{array}{l}0.021 \\
(0.0)\end{array}$ & $\begin{array}{c}0.010 \\
(0.0)\end{array}$ & $\begin{array}{c}0.256 \\
(0.0)\end{array}$ & $\begin{array}{c}3.794 \\
(0.0)\end{array}$ & $\begin{array}{c}0.000 \\
(0.0)\end{array}$ & $\begin{array}{r}3.155 \\
(0.0)\end{array}$ & $\begin{array}{c}0.161 \\
(0.0)\end{array}$ & $\begin{array}{r}0.339 \\
(0.0)\end{array}$ \\
\hline 45 & $\begin{array}{l}\text { Nemipterus } \\
\text { marginatus }\end{array}$ & $\begin{array}{l}\text { Red filament threadfin } \\
\text { bream }\end{array}$ & 2 & $\begin{array}{c}0.472 \\
(0.0)\end{array}$ & $\begin{array}{c}0.006 \\
(0.0)\end{array}$ & $\begin{array}{c}0.005 \\
(0.0)\end{array}$ & $\begin{array}{c}0.241 \\
(0.0)\end{array}$ & $\begin{array}{r}2.871 \\
(0.0)\end{array}$ & $\begin{array}{c}0.000 \\
(0.0)\end{array}$ & $\begin{array}{r}3.623 \\
(0.0)\end{array}$ & $\begin{array}{c}0.332 \\
(0.0)\end{array}$ & $\begin{array}{r}0.239 \\
(0.0)\end{array}$ \\
\hline \multirow[t]{4}{*}{46} & Nemipterus nemurus & $\begin{array}{l}\text { Redspine threadfin } \\
\text { bream }\end{array}$ & 1 & $\begin{array}{c}0.548 \\
(0.0)\end{array}$ & $\begin{array}{c}0.024 \\
(0.0)\end{array}$ & $\begin{array}{c}0.011 \\
(0.0)\end{array}$ & $\begin{array}{l}0.317 \\
(0.0)\end{array}$ & $\begin{array}{c}3.714 \\
(0.0)\end{array}$ & $\begin{array}{c}0.001 \\
(0.0)\end{array}$ & $\begin{array}{l}3.718 \\
(0.0)\end{array}$ & $\begin{array}{c}0.411 \\
(0.0)\end{array}$ & $\begin{array}{c}0.192 \\
(0.0)\end{array}$ \\
\hline & & Total & 31 & 0.527 & 0.013 & 0.012 & 0.256 & 3.339 & 0.000 & 3.345 & 0.238 & 0.268 \\
\hline & & Total (demersal fish) & 119 & 0.612 & 0.013 & 0.019 & 0.563 & 5.724 & 0.001 & 2.164 & 0.226 & 0.239 \\
\hline & & Overall (marine fish) & 296 & 0.496 & 0.008 & 0.019 & 0.262 & 3.796 & 0.001 & 2.481 & 0.214 & 0.265 \\
\hline
\end{tabular}

IQR: Interquartile range. ND: Not detected.

\subsection{Risk Estimation}

The estimated weekly intake (EWI) was presented in Table 3. The results were expressed as per unit body weight per week (ug/kg b.wt/week). Our results estimated that EWI for Cd consumption from Peninsular Malaysia ranged between 0.01 to $1.42 \mathrm{ug} / \mathrm{kg}$ b.wt/week. The estimated EWI of Cd in the study was far below the established PTWI of FAO/WHO JECFA (7 ug/kg b.wt/week). The calculated EWI of $\mathrm{Pb}$ from consumption of various fish species was between 0.03 and $1.02 \mathrm{ug} / \mathrm{kg}$ b.wt/week which was lower than PTWI guideline for $\mathrm{Pb}(25$ ug/kg b.wt/week). The estimated EWI Cu ranged between 0.69 and $27.65 \mathrm{ug} / \mathrm{kg}$ b.wt/week, Zn ranging from 41.55 to $288.3 \mathrm{ug} / \mathrm{kg}$ b.wt/week and Sn were between 5.12 and $103.27 \mathrm{ug} / \mathrm{kg}$ b.wt/week. The results showed that the calculated EWI was below than established PTWI of Cu (3500 ug/kg b.wt/week), Zn (7000 
ug/kg b.wt/week) and Sn (14,000 ug/kg b.wt/week). Therefore consumption of studied fishes was not considered to pose adverse effects to consumer based on FAO/WHO JECFA guidelines.

Table 3. Estimated weekly intake (EWI) of heavy metals from fish consumption.

\begin{tabular}{|c|c|c|c|c|c|c|c|c|c|c|c|}
\hline \multirow{2}{*}{ No. } & \multirow{2}{*}{ Groups/family/ species } & \multirow{2}{*}{ Common name } & \multicolumn{9}{|c|}{ EWI (ug/kg b.wt/week) } \\
\hline & & & $\mathrm{Se}$ & Cd & $\mathrm{Pb}$ & $\mathrm{Cu}$ & $\mathrm{Zn}$ & $\mathrm{Sb}$ & Sn & $\mathrm{Cr}$ & $\mathrm{Mn}$ \\
\hline \multicolumn{12}{|c|}{ Pelagic fish } \\
\hline \multicolumn{12}{|c|}{ Carangidae (80) } \\
\hline 1. & Selaroides leptolepis & Yellowstripe scad & 7.92 & 0.34 & 0.25 & 9.03 & 130.08 & 0.02 & 32.32 & 3.90 & 2.85 \\
\hline 2. & Selar boops & Oxeye scad & 7.03 & 0.14 & 0.27 & 11.67 & 97.07 & 0.01 & 25.63 & 6.27 & 2.85 \\
\hline 3. & Selar crumenopthalmus & Bigeye scad & 13.33 & 0.52 & 0.30 & 7.70 & 103.75 & 0.00 & 69.73 & 2.06 & 4.14 \\
\hline 4. & Atule mate & Yellowtail scad & 10.60 & 0.13 & 0.03 & 16.69 & 153.32 & 0.01 & 22.77 & 4.76 & 4.97 \\
\hline 5. & Caranx sexfasciatus & Bigeye trevally & 10.80 & 0.23 & 0.35 & 12.60 & 171.45 & 0.01 & 41.22 & 6.06 & 3.57 \\
\hline 6. & Seriola dumerili & Greater amberjack & 6.79 & 0.45 & 0.88 & 13.80 & 95.72 & 0.01 & 53.66 & 8.16 & 4.61 \\
\hline 7. & Decapterus kurroides & Redtail scad & 13.66 & 0.69 & 0.16 & 15.77 & 153.65 & 0.01 & 29.15 & 4.28 & 3.91 \\
\hline 8. & Decapterus muruadsi & Round scad & 14.85 & 0.41 & 0.22 & 16.12 & 146.49 & 0.01 & 34.90 & 2.67 & 3.28 \\
\hline 9. & Decapterus russelli & Slender scad & 18.03 & 0.48 & 0.21 & 23.99 & 132.81 & 0.01 & 40.82 & 4.01 & 4.03 \\
\hline 10. & Decapterus macrosoma & Shortfin scad & 19.42 & 1.42 & 0.31 & 15.84 & 288.33 & 0.09 & 23.80 & 4.67 & 4.94 \\
\hline 11. & Megalaspis cordyla & Torpedo scad & 12.05 & 0.42 & 0.22 & 29.15 & 126.60 & 0.01 & 36.53 & 4.87 & 4.51 \\
\hline \multirow[t]{2}{*}{12.} & Parastromateus niger & Black pomfret & 10.36 & 0.27 & 0.64 & 6.30 & 92.55 & 0.02 & 34.57 & 4.15 & 6.95 \\
\hline & \multicolumn{11}{|l|}{ Scrombidae (97) } \\
\hline 13. & Rastrelliger kanagurta & Indian mackerel & 14.32 & 0.24 & 0.60 & 17.54 & 164.06 & 0.01 & 44.53 & 5.64 & 4.01 \\
\hline 14. & Rastrelliger faughni & Faughn's mackerel & 15.04 & 0.54 & 0.73 & 23.13 & 172.16 & 0.01 & 29.05 & 5.37 & 7.73 \\
\hline 15. & Rastrelliger brachysoma & Indo-Pacific mackerel & 13.12 & 0.19 & 0.61 & 16.39 & 172.59 & 0.01 & 24.70 & 2.98 & 3.19 \\
\hline 16. & Scomber australasicus & Blue mackerel & 16.17 & 0.37 & 0.38 & 17.04 & 117.41 & 0.01 & 38.33 & 3.23 & 3.68 \\
\hline 17. & Scomberomorus guttatus & Indo-Pacific king mackerel & 6.93 & 0.05 & 0.20 & 5.96 & 76.82 & 0.01 & 48.17 & 2.59 & 1.72 \\
\hline 18. & Scomberomorus commerson & $\begin{array}{c}\text { Narrowbarred Spanish } \\
\text { mackerel }\end{array}$ & 9.58 & 0.10 & 0.31 & 5.44 & 84.75 & 0.01 & 24.53 & 4.62 & 2.02 \\
\hline 19. & Gymnosarda unicolor & Dogtooth tuna & 17.95 & 0.24 & 0.35 & 16.91 & 136.02 & 0.01 & 32.77 & 3.95 & 3.67 \\
\hline 20. & Sarda orientalis & Striped bonito & 15.55 & 0.26 & 0.25 & 19.58 & 140.72 & 0.01 & 39.26 & 4.26 & 3.29 \\
\hline 21. & Thunnus tonggol & Longtail tuna & 22.05 & 0.15 & 0.21 & 27.65 & 140.61 & 0.01 & 29.42 & 3.55 & 3.51 \\
\hline 22. & Auxis thazard thazard & Frigate tuna & 16.81 & 0.21 & 0.13 & 18.90 & 125.44 & 0.01 & 17.81 & 2.65 & 2.26 \\
\hline \multirow[t]{3}{*}{23.} & Euthymus affinis & Kawakawa & 14.08 & 0.13 & 0.38 & 17.00 & 172.52 & 0.02 & 23.94 & 5.41 & 2.89 \\
\hline & Demersal fish & & & & & & & & & & \\
\hline & Lutjanidae (24) & & & & & & & & & & \\
\hline 24. & Lutjanus argentimaculatus & Mangrove red snapper & 6.51 & 0.03 & 0.20 & 3.34 & 78.53 & 0.07 & 21.08 & 3.62 & 1.71 \\
\hline 25. & Lutjanus gibbus & Humpback red snapper & 9.28 & 0.04 & 0.20 & 3.30 & 56.89 & 0.01 & 23.55 & 3.66 & 3.32 \\
\hline
\end{tabular}




\section{Continued}

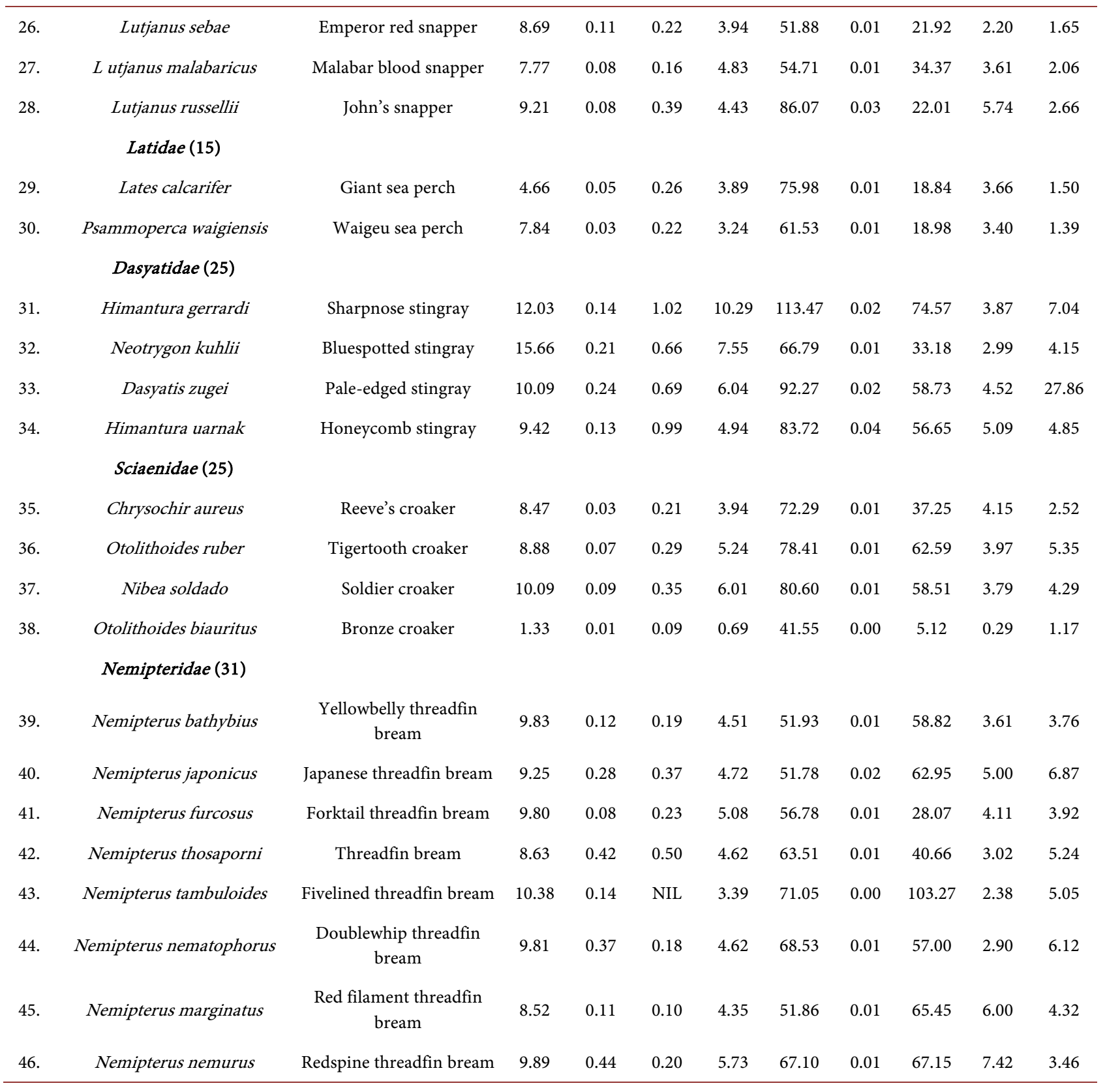

PTWI values for $\mathrm{Cd}, \mathrm{Pb}, \mathrm{Cu}, \mathrm{Zn}$ and $\mathrm{Sn}$ are 7, 25, 3500, 7000 and 14,000 ug/kg b.wt./week (FAO/WHO, 2004; 2011).

Table 4 showed the estimated hazard quotient (HQ) and hazard index (HI) for the consumption of fish for each species. Overall results showed the HQ and HI values were less than one indicated that exposure to the studied metals through ingestion is unlikely to result in adverse health effects. There was a significant discrepancy of HQ among different metals. HQ values of Se were the biggest ranging between 0.0365 (Otolithoides biauritus) and 0.6041 (Thunnus tonggol). Decapterus macrosoma demonstrated the highest HI value (0.8930) compare to other species while Otolithoides biauritus showed the lowest value of HI (0.0593). 
Table 4. Health risk estimates for Se, Cd, $\mathrm{Zn}, \mathrm{Sb}$ and Mn from consumption of marine fish samples in Peninsular Malaysia.

\begin{tabular}{|c|c|c|c|c|c|c|c|c|c|}
\hline \multirow[b]{2}{*}{ No. } & \multirow[b]{2}{*}{ Groups/family/species } & \multirow[b]{2}{*}{ Common name } & \multirow[b]{2}{*}{$\mathbf{n}$} & \multicolumn{5}{|c|}{ HQ } & \multirow{2}{*}{ HI } \\
\hline & & & & $\mathrm{Se}$ & $\mathrm{Cd}$ & $\mathrm{Zn}$ & $\mathrm{Sb}$ & Mn & \\
\hline & Pelagic fish & & & & & & & & \\
\hline & Carangidae (80) & & & & & & & & \\
\hline 1. & Selaroides leptolepis & Yellowstripe scad & 13 & 0.2169 & 0.0468 & 0.0594 & 0.0052 & 0.0028 & 0.3310 \\
\hline 2. & Selar boops & Oxeye scad & 3 & 0.1926 & 0.0190 & 0.0443 & 0.0039 & 0.0028 & 0.2625 \\
\hline 3. & Selar crumenopthalmus & Bigeye scad & 1 & 0.3651 & 0.0711 & 0.0474 & 0.0011 & 0.0041 & 0.4887 \\
\hline 4. & Atule mate & Yellowtail scad & 4 & 0.2905 & 0.0177 & 0.0700 & 0.0026 & 0.0049 & 0.3857 \\
\hline 5. & Caranx sexfasciatus & Bigeye trevally & 1 & 0.2958 & 0.0313 & 0.0783 & 0.0049 & 0.0035 & 0.4138 \\
\hline 6. & Seriola dumerili & Greater amberjack & 1 & 0.1859 & 0.0620 & 0.0437 & 0.0024 & 0.0045 & 0.2986 \\
\hline 7. & Decapterus kurroides & Redtail scad & 4 & 0.3742 & 0.0939 & 0.0702 & 0.0036 & 0.0038 & 0.5457 \\
\hline 8. & Decapterus muruadsi & Round scad & 7 & 0.4069 & 0.0557 & 0.0669 & 0.0028 & 0.0032 & 0.5355 \\
\hline 9. & Decapterus russelli & Slender scad & 10 & 0.4940 & 0.0664 & 0.0606 & 0.0039 & 0.0039 & 0.6289 \\
\hline 10. & Decapterus macrosoma & Shortfin scad & 1 & 0.5320 & 0.1946 & 0.1317 & 0.0299 & 0.0048 & 0.8930 \\
\hline 11. & Megalaspis cordyla & Torpedo scad & 20 & 0.3300 & 0.0571 & 0.0578 & 0.0041 & 0.0044 & 0.4534 \\
\hline \multirow[t]{2}{*}{12.} & Parastromateus niger & Black pomfret & 15 & 0.2838 & 0.0375 & 0.0423 & 0.0068 & 0.0068 & 0.3772 \\
\hline & & Total & 80 & & & & & & \\
\hline & Scrombidae (97) & & & & & & & & \\
\hline 13. & Rastrelliger kanagurta & Indian mackerel & 13 & 0.3924 & 0.0333 & 0.0749 & 0.0039 & 0.0039 & 0.5085 \\
\hline 14. & Rastrelliger faughni & Faughn's mackerel & 6 & 0.4120 & 0.0736 & 0.0786 & 0.0020 & 0.0076 & 0.5738 \\
\hline 15. & Rastrelliger brachysoma & Indo-Pacific mackerel & 3 & 0.3595 & 0.0255 & 0.0788 & 0.0047 & 0.0031 & 0.4716 \\
\hline 16. & Scomber australasicus & Blue mackerel & 18 & 0.4431 & 0.0510 & 0.0536 & 0.0033 & 0.0036 & 0.5546 \\
\hline 17. & Scomberomorus guttatus & Indo-Pacific king mackerel & 12 & 0.1899 & 0.0074 & 0.0351 & 0.0032 & 0.0017 & 0.2374 \\
\hline 18. & Scomberomorus commerson & $\begin{array}{c}\text { Narrowbarred Spanish } \\
\text { mackerel }\end{array}$ & 14 & 0.2625 & 0.0140 & 0.0387 & 0.0041 & 0.0020 & 0.3212 \\
\hline 19. & Gymnosarda unicolor & Dogtooth tuna & 10 & 0.4919 & 0.0334 & 0.0621 & 0.0038 & 0.0036 & 0.5948 \\
\hline 20. & Sarda orientalis & striped bonito & 6 & 0.4260 & 0.0352 & 0.0643 & 0.0035 & 0.0032 & 0.5322 \\
\hline 21. & Thunnus tonggol & Longtail tuna & 8 & 0.6041 & 0.0200 & 0.0642 & 0.0051 & 0.0034 & 0.6968 \\
\hline 22. & Auxis thazard thazard & Frigate tuna & 2 & 0.4606 & 0.0290 & 0.0573 & 0.0019 & 0.0022 & 0.5509 \\
\hline \multirow[t]{4}{*}{23.} & Euthymus affinis & Kawakawa & 5 & 0.3857 & 0.0180 & 0.0788 & 0.0056 & 0.0028 & 0.4909 \\
\hline & & Total & 97 & & & & & & \\
\hline & Demersal fish & & & & & & & & \\
\hline & Lutjanidae (24) & & & & & & & & \\
\hline 24. & Lutjanus argentimaculatus & Mangrove red snapper & 3 & 0.1784 & 0.0045 & 0.0359 & 0.0246 & 0.0017 & 0.2450 \\
\hline 25. & Lutjanus gibbus & Humpback red snapper & 1 & 0.2542 & 0.0055 & 0.0260 & 0.0041 & 0.0032 & 0.2930 \\
\hline 26. & Lutjanus sebae & Emperor red snapper & 11 & 0.2380 & 0.0144 & 0.0237 & 0.0017 & 0.0016 & 0.2795 \\
\hline 27. & L utjanus malabaricus & Malabar blood snapper & 5 & 0.2128 & 0.0109 & 0.0250 & 0.0023 & 0.0020 & 0.2531 \\
\hline \multirow[t]{2}{*}{28.} & Lutjanus russellii & John's snapper & 4 & 0.2524 & 0.0109 & 0.0393 & 0.0118 & 0.0026 & 0.3170 \\
\hline & & Total & 24 & & & & & & \\
\hline
\end{tabular}




\section{Continued}

\begin{tabular}{|c|c|c|c|c|c|c|c|c|c|}
\hline & Latidae (15) & & & & & & & & \\
\hline 29. & Lates calcarifer & Giant sea perch & 11 & 0.1277 & 0.0066 & 0.0347 & 0.0025 & 0.0015 & 0.1730 \\
\hline \multirow[t]{3}{*}{30.} & Psammoperca waigiensis & Waigeu sea perch & 4 & 0.2148 & 0.0047 & 0.0281 & 0.0020 & 0.0014 & 0.2509 \\
\hline & & Total & 15 & & & & & & \\
\hline & Dasyatidae (25) & & & & & & & & \\
\hline 31. & Himantura gerrardi & Sharpnose stingray & 10 & 0.3295 & 0.0187 & 0.0518 & 0.0062 & 0.0069 & 0.4131 \\
\hline 32. & Neotrygon kuhlii & Bluespotted stingray & 7 & 0.4291 & 0.0281 & 0.0305 & 0.0051 & 0.0041 & 0.4969 \\
\hline 33. & Dasyatis zugei & Pale-edged stingray & 5 & 0.2765 & 0.0326 & 0.0421 & 0.0061 & 0.0273 & 0.3845 \\
\hline \multirow[t]{3}{*}{34.} & Himantura uarnak & Honeycomb stingray & 3 & 0.2582 & 0.0172 & 0.0382 & 0.0139 & 0.0047 & 0.3322 \\
\hline & & Total & 25 & & & & & & \\
\hline & Sciaenidae (25) & & & & & & & & \\
\hline 35. & Chrysochir aureus & Reeve's croaker & 3 & 0.2320 & 0.0043 & 0.0330 & 0.0032 & 0.0025 & 0.2751 \\
\hline 36. & Otolithoides ruber & Tigertooth croaker & 6 & 0.2434 & 0.0098 & 0.0358 & 0.0043 & 0.0052 & 0.2985 \\
\hline 37. & Nibea soldado & Soldier croaker & 15 & 0.2764 & 0.0127 & 0.0368 & 0.0040 & 0.0042 & 0.3342 \\
\hline \multirow[t]{3}{*}{38.} & Otolithoides biauritus & Bronze croaker & 1 & 0.0365 & 0.0017 & 0.0190 & 0.0009 & 0.0011 & 0.0593 \\
\hline & & Total & 25 & & & & & & \\
\hline & Nemipteridae (31) & & & & & & & & \\
\hline 39. & Nemipterus bathybius & $\begin{array}{c}\text { Yellowbelly threadfin } \\
\text { bream }\end{array}$ & 6 & 0.2693 & 0.0159 & 0.0237 & 0.0032 & 0.0037 & 0.3157 \\
\hline 40. & Nemipterus japonicus & Japanese threadfin bream & 11 & 0.2533 & 0.0386 & 0.0236 & 0.0055 & 0.0067 & 0.3277 \\
\hline 41. & Nemipterus furcosus & Forktail threadfin bream & 3 & 0.2686 & 0.0104 & 0.0259 & 0.0027 & 0.0038 & 0.3115 \\
\hline 42. & Nemipterus thosaporni & Threadfin bream & 4 & 0.2364 & 0.0572 & 0.0290 & 0.0024 & 0.0051 & 0.3302 \\
\hline 43. & Nemipterus tambuloides & Fivelined threadfin bream & 2 & 0.2845 & 0.0186 & 0.0324 & 0.0012 & 0.0049 & 0.3417 \\
\hline 44. & Nemipterus nematophorus & $\begin{array}{c}\text { Doublewhip threadfin } \\
\text { bream }\end{array}$ & 2 & 0.2688 & 0.0507 & 0.0313 & 0.0028 & 0.0060 & 0.3595 \\
\hline 45. & Nemipterus marginatus & $\begin{array}{l}\text { Red filament threadfin } \\
\text { bream }\end{array}$ & 2 & 0.2335 & 0.0146 & 0.0237 & 0.0024 & 0.0042 & 0.2785 \\
\hline \multirow[t]{2}{*}{46.} & Nemipterus nemurus & Redspine threadfin bream & 1 & 0.2711 & 0.0599 & 0.0306 & 0.0039 & 0.0034 & 0.3689 \\
\hline & & Total & 31 & & & & & & \\
\hline
\end{tabular}

HQ - hazard quotient; HI - hazard index.

Significant variation of heavy metals concentrations, $\mathrm{HQ}$ and $\mathrm{HI}$ values for different factors were presented in Table 5 . The median concentration of $\mathrm{Cu}$, Se, $\mathrm{Cd}$ and $\mathrm{Zn}$ in pelagic fish were significantly higher compared to demersal fish ( $\mathrm{p}$ $<0.05) . \mathrm{Pb}$ and $\mathrm{Mn}$ levels were significantly higher in herbivorous compared to the omnivorous and carnivorous $(\mathrm{Pb}, \mathrm{p}=0.001 ; \mathrm{Mn}, \mathrm{p}<0.05)$. While $\mathrm{Zn}$ level was significantly higher $(\mathrm{p}=0.014)$ in omnivorous compared to other feeding habits. Among family group, $\mathrm{Pb}$ and $\mathrm{Mn}$ levels were found to be higher in $\mathrm{Da}$ syatidae compared to other family groups. Level of Se was higher in Scrombidae; $\mathrm{Cu}, \mathrm{Cd}$ and $\mathrm{Zn}$ levels were higher in Carangidae; Sn level was higher in Sciaenidae compared to other family groups. Meanwhile, $\mathrm{Cr}$ and $\mathrm{Sb}$ levels showed no 
Table 5. Comparison of heavy metals concentration (median; $\mathrm{mg} / \mathrm{kg}$, wet weight) and health risk estimates for marine fish at different factors.

\begin{tabular}{|c|c|c|c|c|c|c|c|c|c|c|c|c|c|c|c|c|}
\hline \multirow{2}{*}{\multicolumn{2}{|c|}{ Factors }} & \multirow{2}{*}{$\frac{\mathrm{Pb}}{\text { median }}$} & \multirow{2}{*}{$\frac{\mathrm{Cu}}{\text { median }}$} & \multirow{2}{*}{$\begin{array}{c}\text { Sn } \\
\text { median }\end{array}$} & \multirow{2}{*}{$\frac{\mathrm{Cr}}{\text { median }}$} & \multicolumn{2}{|c|}{$\mathrm{Se}$} & \multicolumn{2}{|c|}{$\mathrm{Cd}$} & \multicolumn{2}{|c|}{$\mathrm{Zn}$} & \multicolumn{2}{|c|}{$\mathrm{Sb}$} & \multicolumn{2}{|c|}{$\mathrm{Mn}$} & \multirow[t]{2}{*}{ HI } \\
\hline & & & & & & median & HQ & median & HQ & median & HQ & median & HQ & median & HQ & \\
\hline \multirow[t]{4}{*}{ Habitats } & Pelagic & 0.017 & 0.908 & 1.750 & 0.224 & 0.702 & 0.348 & 0.015 & 0.037 & 7.167 & 0.059 & $6.1 \mathrm{E}-04$ & $3.8 \mathrm{E}-03$ & 0.205 & $3.6 \mathrm{E}-03$ & 0.451 \\
\hline & Demersal & 0.018 & 0.269 & 2.278 & 0.203 & 0.511 & 0.253 & 0.006 & 0.016 & 4.019 & 0.033 & $6.5 \mathrm{E}-04$ & $4.0 \mathrm{E}-03$ & 0.217 & $3.8 \mathrm{E}-03$ & 0.309 \\
\hline & p-value & 0.356 & $0.000^{*}$ & 0.103 & 0.133 & $0.000^{*}$ & & $0.000^{*}$ & & $0.000^{*}$ & & $5.5 \mathrm{E}-01$ & & 0.888 & & \\
\hline & Herbivorous & 0.034 & 0.417 & 1.721 & 0.229 & 0.573 & 0.284 & 0.015 & 0.037 & 5.298 & 0.044 & 7.7E-04 & $4.8 \mathrm{E}-03$ & 0.325 & $5.8 \mathrm{E}-03$ & 0.375 \\
\hline \multirow{3}{*}{$\begin{array}{l}\text { Feeding } \\
\text { habits }\end{array}$} & Omnivorous & 0.018 & 0.631 & 1.808 & 0.230 & 0.556 & 0.275 & 0.012 & 0.030 & 6.762 & 0.056 & 7.2E-04 & $4.4 \mathrm{E}-03$ & 0.216 & $3.8 \mathrm{E}-03$ & 0.369 \\
\hline & Carnivorous & 0.016 & 0.625 & 1.865 & 0.204 & 0.594 & 0.294 & 0.009 & 0.023 & 4.951 & 0.041 & $5.7 \mathrm{E}-04$ & $3.5 \mathrm{E}-03$ & 0.193 & $3.4 \mathrm{E}-03$ & 0.364 \\
\hline & $\mathrm{p}$-value & $0.001^{*}$ & 0.722 & 0.471 & 0.186 & 0.887 & & 0.069 & & $0.014^{*}$ & & $1.4 \mathrm{E}-01$ & & $0.000^{*}$ & & \\
\hline \multirow[t]{8}{*}{ Family } & Carangidae & 0.016 & 1.046 & 1.674 & 0.248 & 0.621 & 0.307 & 0.021 & 0.051 & 7.167 & 0.059 & $6.4 \mathrm{E}-04$ & $4.0 \mathrm{E}-03$ & 0.229 & $4.1 \mathrm{E}-03$ & 0.426 \\
\hline & Scrombidae & 0.017 & 0.901 & 1.766 & 0.204 & 0.771 & 0.382 & 0.010 & 0.025 & 6.528 & 0.054 & 5.7E-04 & $3.6 \mathrm{E}-03$ & 0.177 & $3.1 \mathrm{E}-03$ & 0.467 \\
\hline & Lutjanidae & 0.011 & 0.186 & 1.261 & 0.181 & 0.473 & 0.234 & 0.004 & 0.011 & 3.145 & 0.026 & $6.6 \mathrm{E}-04$ & $4.1 \mathrm{E}-03$ & 0.103 & $1.8 \mathrm{E}-03$ & 0.277 \\
\hline & Latidae & 0.012 & 0.191 & 1.013 & 0.197 & 0.294 & 0.145 & 0.002 & 0.006 & 3.848 & 0.032 & $3.2 \mathrm{E}-04$ & $2.0 \mathrm{E}-03$ & 0.080 & $1.4 \mathrm{E}-03$ & 0.186 \\
\hline & Dasyatidae & 0.050 & 0.392 & 2.993 & 0.213 & 0.644 & 0.319 & 0.012 & 0.029 & 5.269 & 0.043 & 9.7E-04 & $6.0 \mathrm{E}-03$ & 0.355 & $6.3 \mathrm{E}-03$ & 0.403 \\
\hline & Sciaenidae & 0.017 & 0.329 & 3.073 & 0.220 & 0.543 & 0.269 & 0.004 & 0.011 & 4.451 & 0.037 & $6.4 \mathrm{E}-04$ & $4.0 \mathrm{E}-03$ & 0.237 & $4.2 \mathrm{E}-03$ & 0.325 \\
\hline & Nemipteridae & 0.011 & 0.279 & 2.755 & 0.223 & 0.516 & 0.256 & 0.008 & 0.020 & 3.236 & 0.027 & $4.5 \mathrm{E}-04$ & $2.8 \mathrm{E}-03$ & 0.275 & $4.9 \mathrm{E}-03$ & 0.310 \\
\hline & p-value & $0.000^{*}$ & $0.000^{*}$ & $0.000^{*}$ & 0.063 & $0.000^{*}$ & & $0.000^{*}$ & & $0.000^{*}$ & & $7.5 \mathrm{E}-02$ & & $0.000^{*}$ & & \\
\hline \multirow[t]{3}{*}{ Origin } & Local & 0.017 & 0.642 & 1.865 & 0.220 & 0.586 & 0.290 & 0.011 & 0.027 & 5.402 & 0.045 & $6.5 \mathrm{E}-04$ & $4.0 \mathrm{E}-03$ & 0.210 & $3.7 \mathrm{E}-03$ & 0.369 \\
\hline & Import & 0.024 & 0.386 & 1.557 & 0.236 & 0.579 & 0.287 & 0.015 & 0.038 & 5.733 & 0.047 & $6.6 \mathrm{E}-04$ & $4.1 \mathrm{E}-03$ & 0.249 & $4.4 \mathrm{E}-03$ & 0.380 \\
\hline & p-value & 0.375 & 0.275 & 0.225 & 0.642 & 0.372 & & 0.528 & & 0.893 & & $9.5 \mathrm{E}-01$ & & 0.118 & & \\
\hline \multirow[t]{4}{*}{ Coastal } & West coast & 0.016 & 0.555 & 1.598 & 0.192 & 0.552 & 0.273 & 0.010 & 0.025 & 5.122 & 0.042 & $6.8 \mathrm{E}-04$ & $4.2 \mathrm{E}-03$ & 0.210 & $3.7 \mathrm{E}-03$ & 0.348 \\
\hline & East coast & 0.018 & 0.563 & 1.921 & 0.226 & 0.624 & 0.309 & 0.011 & 0.027 & 5.698 & 0.047 & $5.5 \mathrm{E}-04$ & $3.4 \mathrm{E}-03$ & 0.204 & $3.6 \mathrm{E}-03$ & 0.390 \\
\hline & South & 0.015 & 0.707 & 2.540 & 0.243 & 0.567 & 0.281 & 0.012 & 0.030 & 6.380 & 0.053 & 5.7E-04 & $3.5 \mathrm{E}-03$ & 0.212 & 3.7E-03 & 0.371 \\
\hline & p-value & 0.380 & 0.780 & 0.414 & $0.002^{*}$ & $0.044^{\star}$ & & 0.320 & & 0.236 & & $0.022^{*}$ & & 0.856 & & \\
\hline \multirow[t]{3}{*}{$\begin{array}{l}\text { Sampling } \\
\text { points }\end{array}$} & $\begin{array}{l}\text { LKIM fish } \\
\text { landing } \\
\text { complexes }\end{array}$ & 0.017 & 0.500 & 1.756 & 0.201 & 0.611 & 0.302 & 0.010 & 0.026 & 5.568 & 0.046 & $6.5 \mathrm{E}-04$ & $4.0 \mathrm{E}-03$ & 0.205 & $3.6 \mathrm{E}-03$ & 0.382 \\
\hline & $\begin{array}{l}\text { Wholesale } \\
\text { wet market }\end{array}$ & 0.018 & 0.646 & 1.864 & 0.226 & 0.562 & 0.278 & 0.010 & 0.026 & 5.273 & 0.043 & $6.3 \mathrm{E}-04$ & $3.9 \mathrm{E}-03$ & 0.209 & 3.7E-03 & 0.355 \\
\hline & p-value & 0.798 & $0.025^{*}$ & $0.000^{*}$ & 0.084 & $0.005^{*}$ & & $0.000^{*}$ & & 0.325 & & $1.0 \mathrm{E}-01$ & & 0.132 & & \\
\hline \multirow[t]{8}{*}{$\begin{array}{l}\text { Sampling } \\
\text { locations }\end{array}$} & Selayang & 0.022 & 0.915 & 1.553 & 0.222 & 0.508 & 0.251 & 0.007 & 0.017 & 5.473 & 0.045 & $6.0 \mathrm{E}-04$ & 3.7E-03 & 0.198 & $3.5 \mathrm{E}-03$ & 0.321 \\
\hline & Klang & 0.017 & 0.333 & 1.520 & 0.209 & 0.358 & 0.177 & 0.002 & 0.005 & 4.361 & 0.036 & $8.2 \mathrm{E}-04$ & $5.1 \mathrm{E}-03$ & 0.134 & $2.4 \mathrm{E}-03$ & 0.225 \\
\hline & Kuala Pari & 0.017 & 0.574 & 1.865 & 0.225 & 0.551 & 0.273 & 0.011 & 0.027 & 4.673 & 0.039 & $5.4 \mathrm{E}-04$ & $3.3 \mathrm{E}-03$ & 0.182 & $3.2 \mathrm{E}-03$ & 0.345 \\
\hline & $\begin{array}{c}\text { Bukit } \\
\text { Mertajam }\end{array}$ & 0.014 & 0.446 & 1.963 & 0.230 & 0.564 & 0.279 & 0.013 & 0.032 & 5.530 & 0.046 & $6.4 \mathrm{E}-04$ & $4.0 \mathrm{E}-03$ & 0.259 & $4.6 \mathrm{E}-03$ & 0.365 \\
\hline & Kuala Perlis & 0.196 & 0.467 & 2.631 & 0.259 & 0.591 & 0.292 & 0.013 & 0.033 & 4.906 & 0.040 & $9.8 \mathrm{E}-04$ & $6.1 \mathrm{E}-03$ & 0.217 & $3.8 \mathrm{E}-03$ & 0.376 \\
\hline & Mergong & 0.018 & 0.836 & 2.693 & 0.229 & 0.660 & 0.326 & 0.014 & 0.035 & 5.950 & 0.049 & $7.6 \mathrm{E}-04$ & $4.7 \mathrm{E}-03$ & 0.263 & 4.7E-03 & 0.420 \\
\hline & Kuala Besar & 0.020 & 0.346 & 1.293 & 0.203 & 0.646 & 0.320 & 0.007 & 0.018 & 6.068 & 0.050 & $6.2 \mathrm{E}-04$ & $3.8 \mathrm{E}-03$ & 0.241 & $4.3 \mathrm{E}-03$ & 0.396 \\
\hline & Pandan & 0.015 & 0.707 & 2.540 & 0.243 & 0.567 & 0.281 & 0.012 & 0.030 & 6.380 & 0.053 & $5.7 \mathrm{E}-04$ & $3.5 \mathrm{E}-03$ & 0.212 & $3.7 \mathrm{E}-03$ & 0.371 \\
\hline
\end{tabular}


Continued

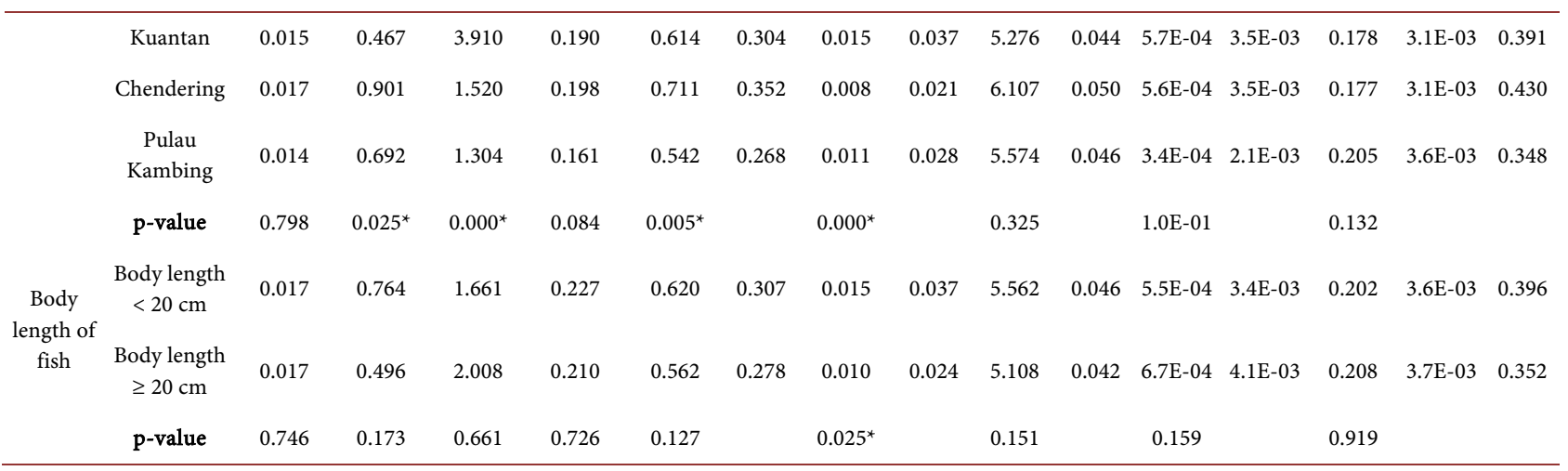

${ }^{*}$ Significant different $(\mathrm{p}<0.05)$.

significant difference between the family group. No significant differences were shown for heavy metals level between local and imported fish $(\mathrm{p}>0.05)$. The coastal region showed significant differences for $\mathrm{Cr}$, Se and Sb levels $(\mathrm{p}<0.05)$. The highest concentrations of $\mathrm{Cr}$ were found on the south coast, Se in east coast while $\mathrm{Sb}$ in the west coast. The concentration of $\mathrm{Cu}, \mathrm{Sn}$, Se and $\mathrm{Cd}$ showed significant different $(\mathrm{p}<0.05)$ between fish landing complexes and wholesale wet market. The levels of $\mathrm{Cu}, \mathrm{Sn}, \mathrm{Se}$ and $\mathrm{Cd}$. Sn and $\mathrm{Cd}$ were found to be higher in Kuantan compared to other sampling location. Cu levels were higher from Selayang, and Se levels were higher from Chendering compared to other locations. Only Cd level was found significantly different $(p=0.025)$ for smaller fish compared to bigger fish.

\section{Discussion}

\subsection{Evaluation of Heavy Metals Concentration in Marine Fishes}

Study on heavy metal in fish has been reported in many studies in Malaysia. However, most of the study focused on the specific location, certain heavy metal and particular fish species. Up to our knowledge, this is first publishing in Malaysia with a high number of samples, representing each state throughout Peninsular Malaysia, and covered nine elements of heavy metals. The results indicated that $\mathrm{Zn}$ was the most abundant metal in the fish samples. Even though $\mathrm{Zn}$ is essential for human, excessive amount could cause a severe health problem. It is quite interesting to discuss on this topic regarding its source, safe daily intake and health risk of zinc exposure through consumption of fish. The data also showed that there were interspecific differences in heavy metals level for all metals. This paper discussed the differences based on several factors, i.e., habitats, feeding habits, family, origin, coastal area, sampling points, sampling location and body length of fish. Each of these factors is discussed below.

Results of this study demonstrated $\mathrm{Zn}$ is the most accumulated metals in fish sample coincided with results reported in several studies conducted in Malaysia [9] [25] [26] [27] [37], which conducted the study in 6 coastal areas in Peninsular Malaysia found that $\mathrm{Zn}$ concentration in fish muscle relatively high among 
the studied elements ( $26.8 \mathrm{ug} / \mathrm{g}$ dry wt.). The study conducted by Bashir et al., 2013 [9] reported $\mathrm{Zn}$ was the highest metal concentration in two fish species, namely Arius thalassinus and Johnius belangeri, ranged between 30.21 to 13.12 ug/g dry weights. Fathi et al., 2013 [37] did a study in the eastern coast of Malaysia, recorded the highest mean concentration of $\mathrm{Zn}$ in Arius thalassinus (35.4 ug/g dry wt.), and the lowest mean concentration of $\mathrm{Zn}$ was in Megalaspis cordyla (17.54 ug/g dry wt.). Zn concentration in Ong et al., 2016 [27] study was lower compared to our study, ranged between $11.172 \mathrm{ug} / \mathrm{g}$ dry wt. (Thunnus sp.) to $5.861 \mathrm{ug} / \mathrm{g}$ dry wt. (Nemipterus sp.). While Kamaruzzaman et al., 2010 [26] study showed $\mathrm{Zn}$ range between $12 \mathrm{ug} / \mathrm{g}$ dry wt. (Selaroides leptolepis) to 25 ug/g dry wt. (Rastrelliger kanagurta) nearly equivalent to our study. The results indicated that bioaccumulation of $\mathrm{Zn}$ from water to muscle of fish is higher compared to other metals [27]. The source of $\mathrm{Zn}$ in natural water mostly from rock weathering process or human activities such as wastes water discharges from industrial. Even though $\mathrm{Zn}$ is an essential element for the human, higher concentration of $\mathrm{Zn}$ could cause health problems such as skin annoyances, stomach cramps, anaemia, vomiting and nausea. High levels of $\mathrm{Zn}$ could also cause damage to the pancreas and disturb the protein metabolism, and cause arteriosclerosis [38]. This study showed that Decapterus macrosoma recorded the highest concentration of $\mathrm{Zn}$ (63.013 ug/g dry wt.) and Cd (0.310 ug/g dry wt.). However, the levels were lower compared to a study conducted by Khalaf et al., 2012 [3] (Zn $94.57 \mathrm{ug} / \mathrm{g}, \mathrm{Cd} 2.32 \mathrm{ug} / \mathrm{g}$ dry wt.). Agusa et al., 2005 [25], reported the much lower result of Zn (29.1 ug/g dry wt.) and Cd (0.162 ug/g dry wt.) in Decapterus macrosoma sample. Elements such as $\mathrm{Pb}, \mathrm{Cd}$, and $\mathrm{Cr}$ were subject to many studies because of their toxicity [25] [39]. $\mathrm{Pb}$ is other known significant contaminants in the environment and occurs naturally in soils, sediments and hydrosphere. $\mathrm{Pb}$ is also widely used in industries which may contribute to pollution in the environment [7]. Cd levels in this study were significantly high in Kuantan area. Kuantan is an urban area and situated near to petrochemical industries, which might contribute to Cd pollution in the coastal area [40]. Burning of fossil fuels and municipal waste are known to be the largest sources of $\mathrm{Cd}$ release to the environment [38].

\subsection{Health Risk Assessment}

Generally, the EWIs obtained from this study did not exceed the standard PTWIs recommended by JEFCA for $\mathrm{Cd}, \mathrm{Pb}, \mathrm{Cu}, \mathrm{Zn}$ and $\mathrm{Sn}$. Nevertheless, the EWI results from our study were higher compared to study conducted by Zaza et al., 2015 [21] in Italy, where EWI for $\mathrm{Cd}$ and $\mathrm{Pb}$ were $0.33 \mathrm{ug} / \mathrm{kg} /$ week and $0.49 \mathrm{ug} / \mathrm{kg} /$ week respectively. The study by Peycheva et al., 2016 [20] in Bulgaria reported that the EWI of $\mathrm{Cu}$ were ranged between 0.480 to $1.279 \mathrm{ug} / \mathrm{kg} /$ week, which is about $25 \%$ lower than our study. While the EWI of $\mathrm{Zn}$ demonstrates significantly lower than our study (7.334 - $15.983 \mathrm{ug} / \mathrm{kg} /$ week). The calculated HQ and HI in this study demonstrated that HQ values were lower than 1, which 
implied that fish consumption from Peninsular Malaysia has low non-cancer risk towards the human. Similar to the findings by Storelli et al., 2008 [2] showed HQ values lower than 1 for $\mathrm{Cd}$. However, the values ranges were much lower compared to our study (HQ; 0.01 to 0.04). The study by Peycheva et al., 2016 [20] also demonstrated that their HQ values for $\mathrm{Zn}$ (0.0005 to 0.0010) were lower compared to our study. Although the values of HQ and HI were much lesser than 1, the consumer should consider the daily intake of fish to ensure safe consumption of fish.

\section{Conclusion}

This study assessed the concentration of nine heavy metals in 46 species of commonly consumed marine fish in Peninsular Malaysia. The obtained data provided comprehensive information as a baseline reference for future studies concerning heavy metals contaminations in marine fish for the country. The results show that different heavy metals demonstrate different accumulation rates in different species. Further study on physiological and ecological factors is suggested to better understand the factors affecting the accumulation of heavy metal in fish species. Health risk assessment shows that the values for HQ and HI were lower than one, suggesting that these pollutants possibly pose a low non-cancer risk to the population. Even though the EWI of the population was lower than PTWI levels, the excessive consumption of fish could lead to adverse effects on human health. It is recommended that regular monitoring of heavy metal contamination of fish species should be carried out to ascertain the safety of consumption of fish.

\section{Acknowledgements}

The authors are grateful to the support and assistance provided by the staff of the Environmental Health Research Centre, Institute for Medical Research. The authors also acknowledge the Fisheries Development Authority of Malaysia and the Malaysian Fisheries Society, for facilitating the sampling process. We would like to thank the Director General of Health Malaysia for his permission to publish this article. This study was funded by the Ministry of Health Malaysia, NMRR ID: NMRR-08-322-1477 (JPPIMR-07-025).

\section{Conflicts of Interest}

The authors declare no conflicts of interest regarding the publication of this paper.

\section{References}

[1] Burger, J. and Gochfeld, M. (2005) Heavy Metals in Commercial Fish in New Jersey. Environmental Research, 99, 403-412. https://doi.org/10.1016/j.envres.2005.02.001

[2] Storelli, M.M. (2008) Potential Human Health Risks from Metals (Hg, Cd, and Pb) and Polychlorinated Biphenyls (PCBs) via Seafood Consumption: Estimation of Target Hazard Quotients (THQs) and Toxic Equivalents (TEQs). Food and Chemi- 
cal Toxicology, 46, 2782-2788. https://doi.org/10.1016/j.fct.2008.05.011

[3] Khalaf, M.A., Al-Najjar, T., Alawi, M. and Disi, A.A. (2012) Levels of Trace Metals in Three Fish Species Decapterus macrellus, Decapterus macrosoms and Decapterus russelli of the Family Carangidae from the Gulf of Aqaba, Red Sea, Jordan. Natural Sciences, 4, 362-367. https://doi.org/10.4236/ns.2012.46050

[4] Zhu, F., Qu, L., Fan, W., Wang, A., Hao, H., Li, X. and Yao, S. (2015) Study on Heavy Metal Levels and Its Health Risk Assessment in Some Edible Fishes from Nansi Lake, China. Environmental Monitoring and Assessment, 187, 161. https://doi.org/10.1007/s10661-015-4355-3

[5] Norimah, A.K., Safiah, M., Jamal, K., Haslinda, S., Zuhaida, H., Rohida, S., Fatimah, S., Azlin, S., Poh, B.K., Kandiah, M., Zalilah, M.S., Wan Manan, W.M., Fatimah, S. and Azmi, M.Y. (2008) Food Consumption Patterns: Findings from the Malaysian Adult Nutrition Survey (MANS). Malaysian Journal of Nutrition, 14, 25-39.

[6] Ahmad, N.I., Noh, M.F.M., Mahiyuddin, W.R.W., Jaafar, H., Ishak, I., Azmi, W.N.F.W., Veloo, Y. and Hairi, M.H. (2015) Mercury Levels of Marine Fish Commonly Consumed in Peninsular Malaysia. Environmental Science and Pollution Research International, 22, 3672-3686. https://doi.org/10.1007/s11356-014-3538-8

[7] Bosch, A.C., O’Neill, B., Sigge, G.O., Kerwath, S.E. and Hoffman, L.C. (2016) Heavy Metals in Marine Fish Meat and Consumer Health: A Review. Journal of the Science of Food and Agriculture, 96, 32-48. https://doi.org/10.1002/jsfa.7360

[8] Ong, M.C. and Kamaruzzaman, B.Y. (2009) An Assessment of Metals ( $\mathrm{Pb}$ and $\mathrm{Cu}$ ) Contamination in Bottom Sediment from South China Sea Coastal Waters, Malaysia. American Journal of Applied Sciences, 6, 1418-1423. https://doi.org/10.3844/ajassp.2009.1418.1423

[9] Bashir, F.H., Othman, M.S., Mazlan, A.G., Rahim, S.M. and Simon, K.D. (2013) Heavy Metal Concentration in Fishes from the Coastal Waters of Kapar and Mersing, Malaysia. Turkish Journal of Fisheries and Aquatic Sciences, 13, 375-382. https://doi.org/10.4194/1303-2712-v13 221

[10] Nor Hasyimah, A.K., James Noik, V., Teh, Y.Y., Lee, C.Y. and Pearline Ng, H.C. (2011) Assessment of Cadmium (Cd) and Lead (Pb) Levels in Commercial Marine Fish Organs between Wet Markets and Supermarkets in Klang Valley, Malaysia. International Food Research Journal, 18, 795-802.

[11] Agusa, T., Kunito, T., Sudaryanto, A., Monirith, I., Supawat, K.A., Iwata, H., Ismail, A., Sanguansin, J., Muchtar, M., Tana, T.S. and Tanabe, S. (2007) Exposure Assessment for Trace Elements from Consumption of Marine Fish in Southeast Asia. Environmental Pollution, 145, 766-777. https://doi.org/10.1016/j.envpol.2006.04.034

[12] Chen, M.-H. and Chen, C.-Y. (1999) Bioaccumulation of Sediment-Bound Heavy Metals in Grey Mullet, Liza macrolepis. Marine Pollution Bulletin, 39, 239-244. https://doi.org/10.1016/S0025-326X(99)00027-2

[13] Canli, M. and Atli., G. (2003) The Relationships between Heavy Metal (Cd, Cr, Cu, $\mathrm{Fe}, \mathrm{Pb}, \mathrm{Zn}$ ) Levels and the Size of Six Mediterranean Fish Species. Environmental Pollution, 121, 129-136. https://doi.org/10.1016/S0269-7491(02)00194-X

[14] Kumar, B., Sajwan, K.S. and Mukherjee, D.P. (2012) Distribution of Heavy Metals in Valuable Coastal Fishes from North East Coast of India. Turkish Journal of Fisheries and Aquatic Sciences, 12, 81-88.

[15] Pintaeva, E.T., Bazarsadueva, S.V., Radnaeva, L.D., Pertov, E.A. and Smirnova, O.G. (2011) Content and Character of Metal Accumulation in Fish of the Kichera River: A Tributary of Lake of Baikal. Contemporary Problems of Ecology, 4, 64-68. https://doi.org/10.1134/S1995425511010103 
[16] Virtanen, J.K., Rissanen, T.H., Voutilainen, S. and Tuomainen, T.P. (2007) Mercury as a Risk Factor for Cardiovascular Diseases. The Journal of Nutritional Biochemistry, 18, 75-85. https://doi.org/10.1016/j.jnutbio.2006.05.001

[17] Raja, P., Veerasingam, S., Suresh, G., Marichamy, G. and Venkatachalapathy, R. (2009) Heavy Metals Concentration in Four Commercially Valuable Marine Edible Fish Species from Parangipettai Coast, South East Coast of India. Journal of Animal and Veterinary Advances, 1, 10-14.

[18] Alinnor, I.J. and Obiji, I.A. (2010) Assessment of Trace Metal Composition in Fish Samples from Nworie River. Pakistan Journal of Nutrition, 9, 81-85. https://doi.org/10.3923/pjn.2010.81.85

[19] WHO (2004) Evaluation of Certain Food Additives and Contaminants. Sixty-First Report of the Joint FAO/WHO Expert Committee on Food Additives.

[20] Peycheva, K., Panayotova, V. and Stancheva, M. (2016) Assessment of Human Health Risk for Copper, Arsenic, Zinc, Nickel, and Mercury in Marine Fish Species Collected from Bulgarian Black Sea Coast. International Journal of Fisheries and Aquatic Studies, 4, 41-46.

[21] Zaza, S., de Balogh, K., Palmery, M., Pastorelli, A.A. and Stacchini, P. (2015) Human Exposure in Italy to Lead, Cadmium and Mercury through Fish and Seafood Product Consumption from Eastern Central Atlantic Fishing Area Silvia. Journal of Food Composition and Analysis, 40, 148-153. https://doi.org/10.1016/j.jfca.2015.01.007

[22] US Environmental Protection Agency (USEPA) (2000) Guidance for Assessing Chemical Contaminant Data for Use in Fish Advisory Vol. II: Risk Assessment and Fish Consumption Limits. US Environmental Protection Agency, Office of Science and Technology, Office of Water, Washington DC, EPA823-B-00-008.

[23] Yap, C.K., Ismail, A., Tan, S.G. and Omar, H. (2002) Correlations between Speciation of $\mathrm{Cd}, \mathrm{Cu}, \mathrm{Pb}$ and $\mathrm{Zn}$ in Sediment and Their Concentrations in Total Soft Tissue of Green-Lipped Mussel Perna viridis from the West Coast of Peninsular Malaysia. Environment International, 28, 117-126. https://doi.org/10.1016/S0160-4120(02)00015-6

[24] Yap, C.K., Ismail, A. and Tan, S.G. (2004) Heavy Metal (Cd, Cu, Pb and Zn) Concentration in the Green-Lipped Mussel Perna viridis (Linnaeus) Collected from Some Wild and Aquaculture Sites in the West Coast of Peninsular Malaysia. Food Chemistry, 84, 569-575. https://doi.org/10.1016/S0308-8146(03)00280-2

[25] Agusa, T., Kunito, T., Yasunaga, G., Iwata, H., Subramanian, A., Ismail, A. and Tanabe, S. (2005) Concentration of Trace Elements in Marine Fish and Its Risk Assessment in Malaysia. Marine Pollution Bulletin, 51, 896-911.

https://doi.org/10.1016/j.marpolbul.2005.06.007

[26] Kamaruzzaman, B.Y., Ong, C. and Rina, S.Z. (2010) Concentration of Zn, Cu and $\mathrm{Pb}$ in Some Selected Marine Fishes of the Pahang Coastal Waters, Malaysia. American Journal of Applied Sciences, 7, 309-314. https://doi.org/10.3844/ajassp.2010.309.314

[27] Ong, M.C., Kamaruzaman, M.I., Siti Norhidayah, A. and Joseph, B. (2016) Trace Metal in Highly Commercial Fishes Caught along Coastal Water of Setiu, Terengganu, Malaysia. International Journal of Applied Chemistry, 12, 773-784.

[28] Ahmad, N.I., Noh, M.F.M., Mahiyuddin, W.R.W., Jaafar, H., Ishak, I., Azmi, W.N.F.W., Veloo, Y. and Mokhtar, F.A. (2015) The Mercury Levels in Crustaceans and Cephalopods from Peninsular Malaysia. Environmental Science and Pollution Research International, 22, 12960-12974. 
https://doi.org/10.1007/s11356-015-4415-9

[29] Tee, E.S., Mohd Ismail, N., Mohd Nasir, A. and Khatijah, I. (1997) Nutrient Composition of Malaysian Foods, ASEAN Sub-Committee on Protein: Food Habits Research and Development. Institute for Medical Research, Kuala Lumpur.

[30] Nurnadia, A.A., Azrina, A. and Amin, I. (2011) Proximate Composition and Energetic Value of Selected Marine Fish and Shellfish from the West Coast of Peninsular Malaysia. International Food Research Journal, 18, 137-148.

[31] Chouvelon, T., Warnau, M., Churlaud, C. and Bustamante, P. (2009) Hg Concentrations and Related Risk Assessment in Coral Reef Crustaceans, Molluscs and Fish from New Caledonia. Environmental Pollution, 157, 331-340. https://doi.org/10.1016/j.envpol.2008.06.027

[32] FAO Food and Agriculture Organization (2009) Fishery and Aquaculture Statistics. http://www.fao.org/3/aq187t/aq187t.pdf

[33] Idriss, A.A. and Ahmad, A.K. (2015) Heavy Metal Concentrations in Fishes from Juru River, Estimation of the Health Risk. Bulletin of Environmental Contamination and Toxicology, 94, 204-208. https://doi.org/10.1007/s00128-014-1452-x

[34] Ahmad, N.I., Mahiyuddin, W.R.W., Mohamad, T.R.T., Ling, C.Y., Daud, S.F., Hussein, N.C., Abdullah, N.A., Shaharudin, R. and Sulaiman, L.H. (2016) Fish Consumption Pattern among Adults of Different Ethnics in Peninsular Malaysia. Food \& Nutrition Research, 60, 32697. https://doi.org/10.3402/fnr.v60.32697

[35] Chien, L.C., Hung, T.C., Choang, K.Y., Yeh, C.Y., Meng, P.J., Shieh, M.J. and Han, B.C. (2002) Daily Intake of TBT, Cu, $\mathrm{Zn}, \mathrm{Cd}$ and As for Fishermen in Taiwan. Science of the Total Environment, 285, 177-185. https://doi.org/10.1016/S0048-9697(01)00916-0

[36] Yi, Y., Tang, C., Yi, T., Yang, Z. and Zhang, S. (2017) Health Risk Assessment of Heavy Metals in Fish and Accumulation Patterns in Food Web in the Upper Yangtze River, China. Ecotoxicology and Environmental Safety, 145, 295-302. https://doi.org/10.1016/j.ecoenv.2017.07.022

[37] Fathi, H.B., Othman, M.S., Mazlan, A.G., Arshad, A., Amin, S.M.N. and Simon, K.D. (2013) Trace Metals in Muscle, Liver and Gill Tissues of Marine Fishes from Mersing, Eastern Coast of Peninsular Malaysia: Concentration and Assessment of Human Health Risk. Asian Journal of Animal and Veterinary Advances, 8, 227-236. https://doi.org/10.3923/ajava.2013.227.236

[38] Afshan, S., Ali, S., Ameen, U.S., Farid, M., Bharwana, S.A., Hannan, F. and Ahmad, R. (2014) Effect of Different Heavy Metal Pollution on Fish. Research Journal of Chemical and Environmental Sciences, 2, 74-79.

[39] Alina, M., Azrina, A., Mohd Yunus, A.S., Mohd Zakiuddin, S., Mohd Izuan Effendi, H. and Muhammad Rizal, R. (2012) Heavy Metals (Mercury, Arsenic, Cadmium, Plumbum) in Selected Marine Fish and Shellfish along the Straits of Malacca. International Food Research Journal, 19, 135-140.

[40] Sujaul, I.M., Hossain, M.A., Nasly, M.A. and Sobahan, M.A. (2013) Effect of Industrial Pollution on the Spatial Variation of Surface Water Quality. American Journal of Environmental Sciences, 9, 120-129. https://doi.org/10.3844/ajessp.2013.120.129 